San Jose State University

SJSU ScholarWorks

Master's Projects

Master's Theses and Graduate Research

Spring 5-20-2019

\title{
JavaScript Metamorphic Malware Detection Using Machine Learning Techniques
}

Aakash Wadhwani

San Jose State University

Follow this and additional works at: https://scholarworks.sjsu.edu/etd_projects

Part of the Artificial Intelligence and Robotics Commons, and the Information Security Commons

\section{Recommended Citation}

Wadhwani, Aakash, "JavaScript Metamorphic Malware Detection Using Machine Learning Techniques" (2019). Master's Projects. 688.

DOI: https://doi.org/10.31979/etd.8rtn-buzk

https://scholarworks.sjsu.edu/etd_projects/688

This Master's Project is brought to you for free and open access by the Master's Theses and Graduate Research at SJSU ScholarWorks. It has been accepted for inclusion in Master's Projects by an authorized administrator of SJSU ScholarWorks. For more information, please contact scholarworks@sjsu.edu. 
JavaScript Metamorphic Malware Detection Using Machine Learning Techniques

\author{
A Project \\ Presented to \\ The Faculty of the Department of Computer Science \\ San José State University
}

In Partial Fulfillment

of the Requirements for the Degree

Master of Science

by

Aakash Wadhwani

May 2019 
(C) 2019

Aakash Wadhwani

ALL RIGHTS RESERVED 
The Designated Project Committee Approves the Project Titled

JavaScript Metamorphic Malware Detection Using Machine Learning Techniques

by

Aakash Wadhwani

APPROVED FOR THE DEPARTMENT OF COMPUTER SCIENCE

SAN JOSÉ STATE UNIVERSITY

May 2019

Dr. Thomas Austin Department of Computer Science

Prof. Mark Stamp Department of Computer Science

Prof. Philip Heller Department of Computer Science 


\begin{abstract}
JavaScript Metamorphic Malware Detection Using Machine Learning Techniques by Aakash Wadhwani
\end{abstract}

Various factors like defects in the operating system, email attachments from unknown sources, downloading and installing a software from non-trusted sites make computers vulnerable to malware attacks. Current antivirus techniques lack the ability to detect metamorphic viruses, which vary the internal structure of the original malware code across various versions, but still have the exact same behavior throughout. Antivirus software typically relies on signature detection for identifying a virus, but code morphing evades signature detection quite effectively.

JavaScript is used to generate metamorphic malware by changing the code's Abstract Syntax Tree without changing the actual functionality, making it very difficult to detect by antivirus software. As JavaScript is prevalent almost everywhere, it becomes an ideal candidate language for spreading malware.

This research aims to detect metamorphic malware using various machine learning models like K Nearest Neighbors, Random Forest, Support Vector Machine, and Naïve Bayes. It also aims to test the effectiveness of various morphing techniques that can be used to reduce the accuracy of the classification model. Thus, this involves improvement on both fronts of generation and detection of the malware helping antivirus software detect morphed codes with better accuracy. In this research, JavaScript based metamorphic engine reduces the accuracy of a trained malware detector. While N-gram frequency based feature vectors give good accuracy results for classifying metamorphic malware, HMM feature vectors provide the best results. 


\section{ACKNOWLEDGMENTS}

I would like to thank my project advisor Prof. Thomas Austin. Without his constant support and guidance, this project would not have been possible.

I would also like to thank my committee members Prof. Mark Stamp and Prof. Philip Heller for monitoring my progress throughout and guiding me with their expertise in the field of Machine Learning and Information Security. 


\section{TABLE OF CONTENTS}

\section{CHAPTER}

1 Introduction . . . . . . . . . . . . . . . . . 1

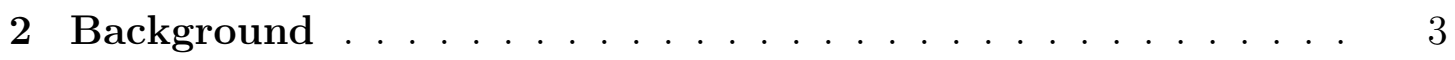

2.1 Malware ........................ . . . 3

2.2 Malware Types . . . . . . . . . . . . . . . . . . 4

2.2 .1 Virus . . . . . . . . . . . . . . . . 4

2.2 .2 Worms ....................... 5

$2.2 .3 \quad \operatorname{Trojan} \ldots \ldots \ldots \ldots \ldots \ldots \ldots \ldots \ldots$

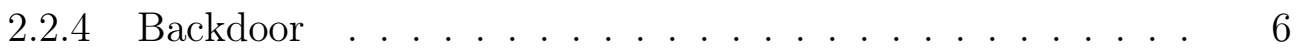

2.3 Detection Techniques . . . . . . . . . . . . . 6

2.3.1 Signature Detection . . . . . . . . . . . . . 6

2.3.2 Behavioral Detection . . . . . . . . . . . . . 7

2.3 .3 Anomaly Detection . . . . . . . . . . . . . 7

2.3.4 Hidden Markov Model (HMM) . . . . . . . . . . . 8

3 Related Work . . . . . . . . . . . . . . . . . . . 11

4 Morphing Engine . . . . . . . . . . . . . . . . . . . . . 12

4.1 Abstract Syntax Tree . . . . . . . . . . . . . . . . . . . 12

4.2 Engine Implementation Details _ . . . . . . . . . . . . . 14

4.3 Architecture Overview . . . . . . . . . . . . . . . . . 15

4.4 Techniques Used $\ldots \ldots \ldots \ldots$

4.4 .1 Variable Renaming . . . . . . . . . . . . . . 16 
4.4 .2 Dead Code Insertion . . . . . . . . . . . . . . 17

4.4 .3 Instruction Reordering . . . . . . . . . . . . . . . . 17

4.4.4 Function Reordering . . . . . . . . . . . . . . 18

4.4.5 Instruction Substitution $\ldots \ldots \ldots \ldots$

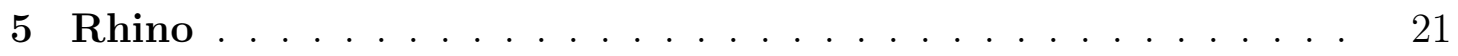

5.1 Architecture . . . . . . . . . . . . . . . . . 21

5.2 Steps . . . . . . . . . . . . . . . . . . . 24

6 Detecting Metamorphic JavaScript f . . . . . . . . . 26

6.1 Dataset . . . . . . . . . . . . . . . . 26

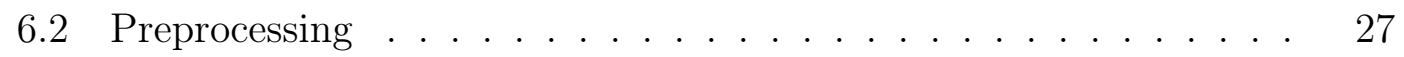

6.3 Feature Extraction $\ldots \ldots \ldots \ldots \ldots \ldots \ldots$

$6.3 .1 \quad \mathrm{~N}$-gram Extraction $\ldots \ldots \ldots \ldots \ldots$

6.3.2 HMM feature extraction . . . . . . . . . . . 30

6.4 Training using N-gram . . . . . . . . . . . . . . 30

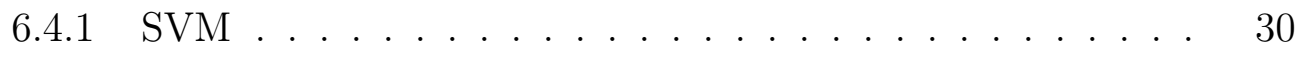

6.4 .2 Random Forest . . . . . . . . . . . . . . . . . . . . . 31

$6.4 .3 \quad$ K Nearest Neighbors . . . . . . . . . . . . . . . . 31

6.4 .4 Naive Bayes . . . . . . . . . . . . . . . . . . . 31

6.5 НMм . . . . . . . . . . . . . . . . . . 31

6.5 .1 Training . . . . . . . . . . . . 32

6.5.2 Classification using HMM vectors . . . . . . . . . . 32

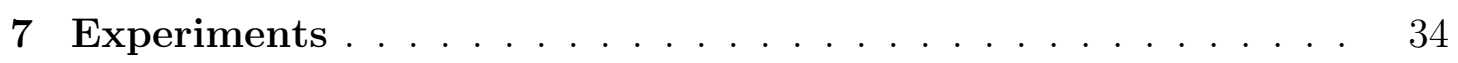

$7.1 \quad$ Setup . . . . . . . . . . . . . . . . . . 34 
7.2 Results . . . . . . . . . . . . . . .

7.2.1 Reducing the accuracy of malware detector . . . . . . . 35

7.2 .2 Building a metamorphic detector $\ldots \ldots \ldots \ldots$

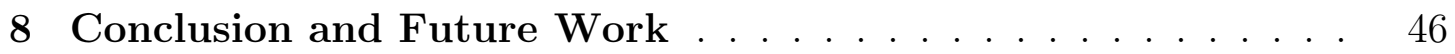

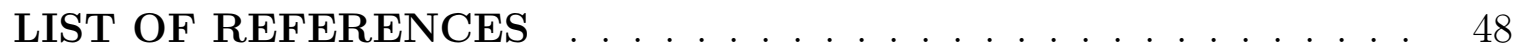

\section{APPENDIX}

Appendix A . . . . . . . . . . . . . . . . . . 52

A.1 HMM for detecting metamorphic malware with $\mathrm{N}=3, \mathrm{M}=26$,

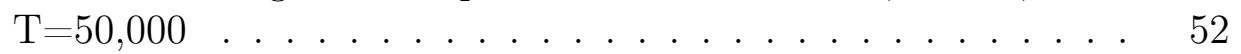

A.2 HMM for detecting metamorphic malware with $\mathrm{N}=5, \mathrm{M}=26$,

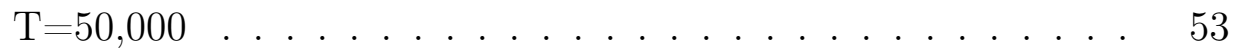

A.3 K-Nearest Neighbors for metamorphic malware detection using $\mathrm{N}$-gram features with $\mathrm{K}=[3,7,11] \ldots \ldots \ldots \ldots$

A.4 Random Forest for metamorphic malware detection using N-gram features with Trees $=[200,300] \ldots \ldots \ldots \ldots$ 


\section{LIST OF TABLES}

1 HMM Notations [1] ..................... 8

2 Host Machine Specifications . . . . . . . . . . . . . . 34

3 Guest Machine Specifications . . . . . . . . . . . . . 35

$4 \quad$ Accuracy Drop . . . . . . . . . . . . . . . . 38 


\section{LIST OF FIGURES}

1 New Malware programs in last 10 years [2] . . . . . . . . 3

2 Generic view of Hidden Markov Model [1] . . . . . . . . . . . 9

3 Abstract Syntax Tree using esprima . . . . . . . . . . . . 13

4 Morphing Engine Architecture . . . . . . . . . . . . . 15

5 Rhino Block Diagram [3] . . . . . . . . . . . . . . . 22

$6 \quad$ Abstract Syntax Tree [3] . . . . . . . . . . . . . 23

7 rhinoFile.js Bytecode . . . . . . . . . . . . 25

$8 \quad$ Example sequence file . . . . . . . . . . . . . . . 28

9 Random Forest . . . . . . . . . . . . . . . . . 36

$10 \quad$ K Nearest Neighbours _. . . . . . . . . . . . . . . . . . 37

$11 \quad$ Support Vector Machine . . . . . . . . . . . . . . . . 37

$12 \quad$ Naïve Bayes . . . . . . . . . . . . . . . . . . . 38

13 Accuracy Drop Graph . . . . . . . . . . . . . . . 39

14 Accuracy Drop - Dead Code . . . . . . . . . . . . . . 40

15 Random Forest using N-grams . . . . . . . . . . . . . 41

$16 \quad$ K Nearest Neighbours using N-grams . . . . . . . . . . . . . . . . 41

17 Support Vector Machine using N-grams . . . . . . . . . . . 42

18 Naive Bayes using N-grams . . . . . . . . . . . . . 42

19 Random Forest using HMM vectors . . . . . . . . . . . . . . 43

20 K Nearest Neighbours using HMM vectors . . . . . . . . . . . . 44

21 Support Vector Machine using HMM vectors . . . . . . . . . . . 44 
22 Naive Bayes using HMM vectors . . . . . . . . . . . . . . 45

A.23 HMM with $\mathrm{N}=3, \mathrm{M}=26, \mathrm{~T}=50,000 \ldots \ldots . \ldots . \ldots 2$

A.24 HMM with $\mathrm{N}=5, \mathrm{M}=26, \mathrm{~T}=50,000 \ldots \ldots . \ldots 53$

A.25 N-grams with K-Nearest Neighbor (KNN) as classifier . . . . . 54

A.26 N-grams with Random Forest as classifier . . . . . . . . 55 


\section{CHAPTER 1}

\section{Introduction}

Malware is computer software that is developed with the intention of infiltrating a computer system without the user's consent. The main purpose of a malware is to extract critical information from one's system and access confidential resources without a user's permission. This malware can delete files from a user's computer and infect them with malicious code, thereby stealing private information from a user

or causing a computer to cease to work normally. These codes stop the execution of non-malicious code or run beside them to harm the host computer.

There are millions of services and web applications on the web, such as social networking websites, content distribution websites, entertainment, and academic sites making the internet a core part of our daily lives. With its ubiquity and popularity, the web has become an integral platform for malware creators. Malware seeks to evade security mechanisms by performing activities like getting access to the user's computer and its resources, delaying services provided by users through denial of service(DoS) attacks, and unwanted spamming. It becomes very difficult for the end user to protect their system from malware, since most of them lack the expertise. There is no central authority to ensure security as the web is a vast distributed system.

While there are few ways to introduce malware using HTML, Javascript enables the malware writers to download and run malicious code by making the victim browse any website. Bugs in Javascript or incorrect implementations could introduce backdoors which can be exploited by attackers. The ubiquitous nature of JavaScript across the web makes the users vulnerable to many attacks. Even a small amount of Javascript code inside a HTML file is enough to record any activity on a website without having to submit anything. The activities like scrolling, cursor movements, keystrokes can be recorded for the purposes of understanding user's behavior without 
their consent. One of the possibilities of JavaScript is cross-site scripting(XSS). Cross side scripting enables hackers to embed malicious Javascript based code inside a legitimate web page. This will make the end user get affected by malwares even by visiting the trusted websites. Server-side malware involves malware scripts that casuse Denial of Service attacks, backdoors, web-shells, and bruteforce attacks [4].

Over the years, several techniques have been introduced for the detection of this malware. Signature detection is used by various antivirus software which detect the malware based on the physical structure of the virus code. The major problem with this detection technique is that when the virus code is morphed, providing a newer version of the same code, it fails to detect the malware. This limitation motivates the introduction of malware detection using machine learning techniques like K Nearest Neighbors, Support Vector Machine, Random Forest, Naïve Bayes and Hidden Markov Models (HMM). HMM is a statistical model which uses probability distribution amongst the observation sequences containing malware and benign codes to detect malwares.

In this research, we build a malware classifier and analyze various Javascript code morphing techniques to deteriorate the accuracy of the classifier. Later, we experiment on different machine learning techniques to better detect these morphed malware files.

The chapters are organized in the following manner: Chapter 2 gives background on malware and explains why it is important to detect them; Chapter 3 explains the related work on malware detection; Chapter 4 explains the metamorphic engine used to reduce the classification accuracy of a malware detector. Chapter 5 explains the usage of Rhino JavsScript Engine to extract opcode sequences from source code. Chapter 6 explains approaches to detect metamorphic malware files; and chapter 7 explain the experiments carried out to detect metamorphic malware. 


\section{CHAPTER 2}

\section{Background}

\subsection{Malware}

Malware is software written to cause damage to any system on or off the computer network. Much of the earlier malware were written with the intention of pranks or, in some cases Digital Rights Management (DRM). Sony Music Compact discs installed a rootkit on buyers systems for preventing piracy, but this gave access to unauthorized access to user's listening habits leading to security vulnerabilities [5]. A security report by AV-Test [2] found the drastic increase in development of new malware programs from 2007-2017.

\section{Overall development of new malware programs in the last 10 years}

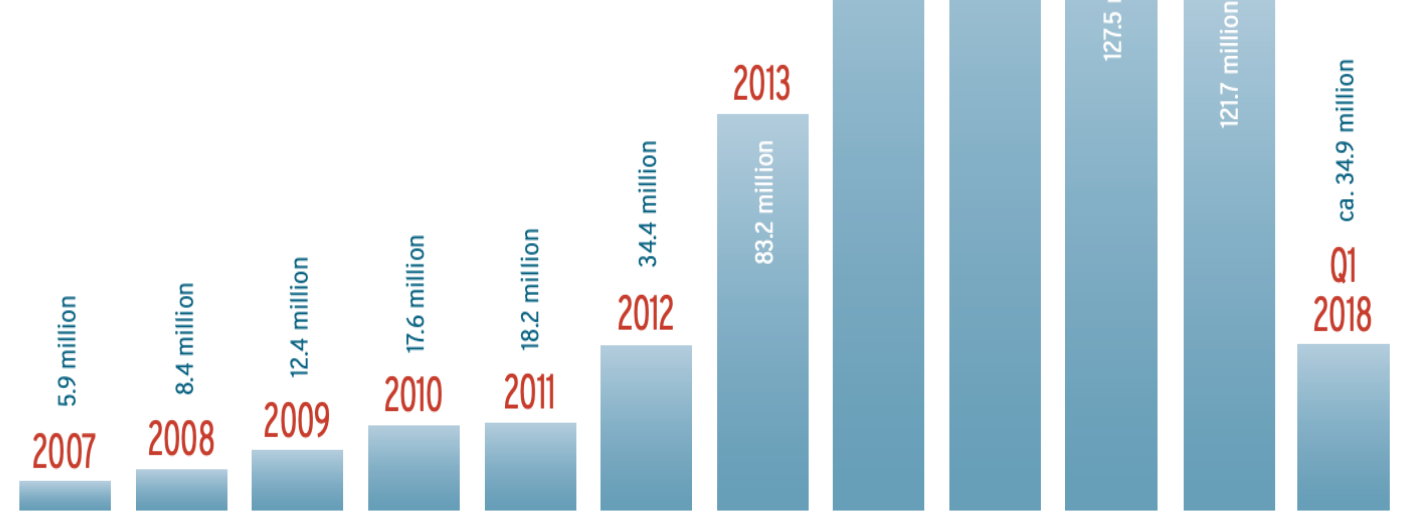

Figure 1: New Malware programs in last 10 years [2]

The $\mathrm{x}$-axis denotes the years from 2007-2018 and the y-axis denotes the number of new malware programs. We can see the spike in 2014 where the number increased from 83.2 million to 143.1 million. Thus, there is an aggressive need to solve malware detection problem with such a huge rate in increase of new malware programs. 


\subsection{Malware Types}

Malware is a superset of all kinds of viruses families, Trojans, backdoor virus, adware, worms, etc [6].

\subsubsection{Virus}

A virus is a type of malware which has the property of self-replication to spread itself to other executable programs [7]. In turn, these affected executable programs spread the infection to other benign files. The virus can live anywhere in the system like in the executable files, data files, hidden files or even the boot sector. As explained in [8] [7] [9], virus can exist before any antivirus program boots up or any other operating system based security is enabled. Thus, the antivirus would be in no state to prevent virus from infecting the system as even before the antivirus boots up, the virus would have infected the system. The virus can also prevent the antivirus from booting all together. This virus can then be spread across various means like email, external storage devices like hard disk drives, pen drives, floppy drives, etc.

\subsubsection{Encrypted virus}

The initial approach to detect any virus was to store the signature of the virus, in which an instruction sequence or a pattern is stored against each virus so that next time same virus attempts to defect the system it can be detected [7] [9]. To go around this trivial detection mechanism, malware writers introduced encrypted viruses where a malware is encrypted with an encryption technique and key. Thus, signature detection becomes difficult. The problem with this is that the code to decrypt is also attached along with the encrypted malware's body. But, it is still possible to search and store encrypted pattern similar to storing a malware signature. And thus, it can be still detected using signature detection. The decryptor loop is constant in the encrypted virus and hence can be detected easily. Next logical approach [8] is to 
change the decryptor loop with each version of the virus.

\subsubsection{Polymorphic Virus}

The decryptor loop being constant in encrypted virus, makes it easier for detection. There was a need to hide the decryption code and hence the inception of Polymorphic malware [10]. With every version of the virus, the decrypted code is encrypted too [7] [9]. Thus, with infinite possibilities of decryptor loop variations, it makes it very difficult to detect Polymorphic virus by listing all possible combinations of the decryptor loop [8]. Morphing of the decryptor loop is done in such a way that the the code looks completely different but is logically the same [11].

\subsubsection{Metamorphic Virus}

In metamorphic virus, the entire body of the virus is morphed before affecting the system [11] [7] [9]. Thus, it becomes very difficult to detect any metamorphic virus. The appearance of the virus completely changes and hence it is very challenging to detect these types of virus files [12] [13].

\subsubsection{Worms}

A worm is similar to a virus by having a property of self-replication to other computers in the network [14]. The primary difference between a worm and a virus is that the worm does not need a host file to rely on and can be an independent file that damages other files [9] [7]. Worms can replicate rapidly and damage any system by saturating it.

\subsubsection{Trojan}

Unlike a virus, A Trojan does not self-replicate. It acts as a harmless program

in the system. When the user click on these programs, the Trojans install harmful programs in the system causing damage [8]. Trojans could delete files, remove any data from the hard disk drive, open backdoors which act as security vulnerabilities in 
the system[9] [7]. They act as legitimate functions but instead drop malicious payloads into the system.

\subsubsection{Backdoor}

The backdoor is a program that will allow other malicious programs to carry out damage to the system [8]. The other user programs compromise the end users confidential resources [7] [9]. Backdoors allow malicious programs to have damaging consequences on the host computer like deleting all the files, stealing confidential information, it also lets open communication ports allowing other computer systems have remote control over the host computer.

\subsection{Detection Techniques}

In this section, we will look at the various detection techniques based on static analysis like some pattern matching inside malware and dynamic analysis like behaviour of the malware. Most antivirus software depend on signature based detection of malware [15]. The dynamic analysis can be done by models like Hidden Markov Models(HMM).

\subsubsection{Signature Detection}

Signature based detection is the most common technique in antivirus software programs [16]. Signature detection is based on pattern matching, where a sequence of bits against a malware program is stored on a malware database. When the antivirus scans the system it searches for programs having the signatures in the malware database. If any file gets matched with the files in the malware database it is marked as a malware too and hence used later for malware signature matching.

The advantages of using signature based detection is speed and accuracy. With this, the disadvantages of using signature based detection are that it only compares the malware from the malware database. Any new malware files would never be 
detected by such static detection mechanisms. Thus, the malware database needs to be constantly updated. Even a small obfuscation will cause this detection technique to break. Thus, dynamic analysis is required for dynamic behaviors of the virus.

\subsubsection{Behavioral Detection}

Behavior based detection works on dynamic behavior of the files instead of the static signature based analysis [17] [18]. The dynamic behavior of the file is extracted by running a file in an emulated environment [7] [19]. The main purpose of this is that even if the virus would look like a legitimate program, its functionality would explain that it is actually a malware program. Behavior analysis along with machine learning is used [20] to classify a file into a malware or a benign file. The emulator would generate a report on each file, explaining the API features involved in the file. These features are then used in the training phase and the files are classified into malware or benign.

\subsubsection{Anomaly Detection}

Anomaly detection works on the approach of detecting if the file given follows a normal behavior [7]. It overcomes the limitations of signature based detection by using heuristic based approaches to detect normal behavior. Any file that doesn't classify as normal is then classified as a malware. The definition of anomalous and normal is user defined, so it doesn't provide a lot of accuracy in classification.

In [21], a malware detector was developed to classify the input files into classes based on structure of the files. A test file which needs classification is given as an input to the detector. The file is classified as anomalous if it looks like one, else it is marked as benign. After classification, it is reviewed to determine if it was a malware file or it was just a false positive case [16]. Anomaly detection might have a lot of false positives or false negatives [16]. This is the reason Anomaly detection is used 
with Signature detection to provide higher accuracy.

\subsubsection{Hidden Markov Model (HMM)}

HMM is a statistical model which depends on the probability distribution of the data and are well suited for statistical pattern analysis [1] [22] [23]. HMM is a state machine which means that it can be in one of the set number of states depending on the previous state and the present value of its input. In HMM, the states are not visible to the user (hidden), hence is a special type of Markov model and consequently named as Hidden Markov Models. In HMM, every state has a probability distribution to observe a set of observation symbols and the transition from one state to another has fixed probabilities. We can match the trained HMM sequence against the test sequence/observation sequence. If the probability is high, we can say that the observation sequence is similar to trained HMM sequence [1]. The following notations in Table 1 are used in HMM [1]:

Table 1: HMM Notations [1]

\begin{tabular}{||c||c||}
\hline Symbol & Definition \\
\hline \hline $\mathrm{T}$ & length of the observation sequence \\
$\mathrm{N}$ & number of states in the model \\
$\mathrm{M}$ & number of distinct observation symbols \\
$\mathrm{Q}$ & distinct states of the Markov Model \\
$\mathrm{V}$ & set of possible observations \\
$\mathrm{A}$ & state transition probability matrix \\
$\mathrm{B}$ & observation probability matrix \\
$\pi$ & initial state distribution \\
$\mathrm{O}$ & observation sequence \\
\hline \hline
\end{tabular}

A HMM is defined by the matrices A, B and $\pi$.

An HMM is denoted as $\lambda=(\mathrm{A}, \mathrm{B}, \pi)$.

The Generic view of Hidden Markov Model [1] is explained in Figure 2 Hidden Markov Model solve three types of problems. 


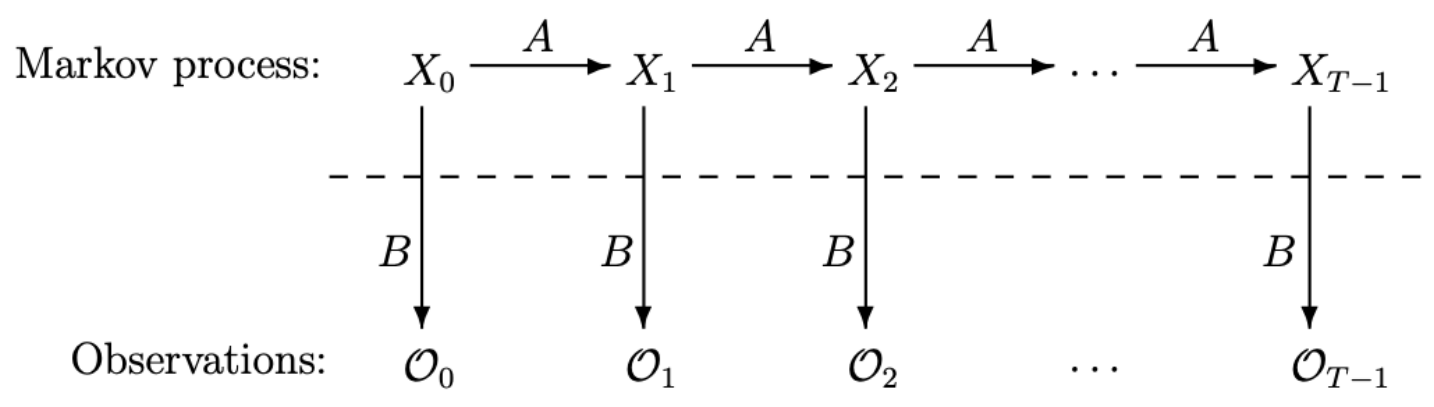

Figure 2: Generic view of Hidden Markov Model [1]

Problem 1: Given a model $\lambda=(\mathrm{A}, \mathrm{B}, \pi)$ and an observation sequence $\mathrm{O}$, we need to find $\mathrm{P}(\mathrm{O} \mid \lambda)$. So, we need to find an observation sequence that fits well to a given model.

Problem 2: Given a model $\lambda=(\mathrm{A}, \mathrm{B}, \pi)$ and an observation sequence $\mathrm{O}$, most likely hidden state sequence can be determined for the model.

Problem 3: Given $\mathrm{O}, \mathrm{N}, \mathrm{M}$, we can find a model $\lambda$ that maximizes probability of O. That is we train the model in order to best fit an observation sequence [1].

In this project, Problem 3 is used. Problem 3 is used when we train the model using the malware source code observation sequence and later files are scored against this model using machine learning classification. If the observation sequence is close to the trained model, we say it is also a malware, otherwise it is not.

Problem 1, Problem 2 and Problem 3 are solved by Forward Algorithm, Backward Algorithm, Baum-Welch re-estimation algorithm.

The forward algorithm determines $\mathrm{P}(\mathrm{O} \mid \lambda)[1]$ which is the probability of being in a state $q_{i}$ given an observation sequence $\mathrm{O}$ [1].

Baum-Welch algorithm is described in [1] [7] and explained as follows: 
1. Initialize $\lambda=(\mathrm{A}, \mathrm{B}, \pi)$ with random values for $\mathrm{A}, \mathrm{B}$ and $\pi$ and making the matrices row stochastic.

2. Compute $\alpha_{t}(i), \beta_{t}(i), \gamma_{t}(i)$ and $\gamma_{t}(i, j)$ Equation for digammas:

$$
\gamma_{t}(i, j)=\frac{\alpha_{t}(i) a_{i, j} b_{j}\left(O_{t+1}\right) \beta_{t+1}(j)}{P(O \mid \lambda)}
$$

3. $\gamma_{t}(i)$ and $\gamma_{t}(i, j)$ relation:

$$
\gamma_{t}(i)=\sum_{j=0}^{N-1} \gamma_{t}(i, j)
$$

4. Re-estimate: For $\mathrm{i}=0,1, \ldots, \mathrm{N}-1$ let

$$
\pi_{i}=\gamma_{0}(i)
$$

5. For $\mathrm{i}=0,1, \ldots, \mathrm{N}-1$ and $\mathrm{j}=0,1, \ldots, \mathrm{N}-1$, compute

$$
a_{i, j}=\sum_{t=0}^{T-2} \gamma_{t}(i, j) / \sum_{t=0}^{T-2} \gamma_{t}(i)
$$

6. For $\mathrm{j}=0,1, \ldots, \mathrm{N}-1$ and $\mathrm{k}=0,1, \ldots, \mathrm{M}-1$, compute

$$
b_{j}(k)=\sum_{t \in(0,1, \ldots . T-2), O_{t}=k} \gamma_{t}(j) / \sum_{t=0}^{T-2} \gamma_{t}(j)
$$

7. for increase in $\mathrm{P}(\mathrm{O} \mid \lambda)$ go to step 3. 


\section{CHAPTER 3}

\section{Related Work}

Machine Learning has been long involved in malware detection. Naïve Bayes is a model that works on the likelihood of certain features in the model. This model is introduced by Schultz et al [24] for malware detection. If certain features occur in a malware, the probability of these featues occurring in any test file is taken and predicted if the given file is a malware. The cons of this approach is that if the features are changed, the model will fail to detect malware files. Heuristic based machine learning is introduced by Bazrafshan et al [15] for malware detection. To overcome the limitation of Naïve Bayes, Hidden Markov Model (HMM) for malware detection is introduced by Rezaei et al [25].

Sridhara [26] explains opcode extraction from a source code as features for the purpose of training an HMM on those opcodes. Hidden Markov Model performs much better as compared to signature-based detection when it comes to unseen malware. As compared to behavior based detection [18], HMM is much faster as it doesn't take time to run the malware to verify its intent. According to Rezaei et al. [25], HMM provides more than $70 \%$ of malware detection which is $15 \%$ more than any other antivirus techniques. 


\section{CHAPTER 4}

\section{Morphing Engine}

The idea of the morphing engine is to morph the Javascript code into another form of code, which would have different physical appearance, but would be logically the same. In this research, we will look at morphing code at the Abstract Syntax Tree (AST) level [27].

\subsection{Abstract Syntax Tree}

Abstract Syntax Tree is a representation of source code in a hierarchical format according to the programming language grammar [28]. Irrespective of the programming language used, the source code needs to parsed as plain text and converted into an Abstract Syntax Tree data structure (AST). The software that parses the plain text and converts it into a logical grammar can be a compiler(C, JAVA, RUST) or an interpreter(JavaScript, Python, Ruby). The role of the Abstract Syntax Tree along with presenting the code in a structured format, is to also help in semantic analysis of the code, resulting in validating the correctness of the language used. ASTs are also used to generate bytecode or machine code going forward. Applications of AST include language transpilation. For example, we could represent a Python based code into its AST and that will be used to generate JavaScript based code or reverse.

In compiler design [29], after tokenization, the parsing phase will take those tokens and generate an Abstract Syntax Tree (AST). It converts the plain text code into a logical structure as understood by the language, and it will skip spaces, new lines, and comments to create that logical structure and hence prefixed as "Abstract". The parser will attempt to match tokens to pre-defined pattern of tokens. For example, assignment $=$ identifier, equals, identifier ;

Given a simple code like in the Listing 4.1, we get the Abstract syntax Tree as given in Figure 3 


\subsection{Engine Implementation Details}

For morphing, certain steps are followed for each source code:

- Convert Code To AST:

The user inputs the JavaScript code which is converted to an abstract syntax tree using the nodeJS module Esprima [30]. The abstract syntax tree is a tree representation of the abstract syntactic structure of the source code. This is useful because it abstracts the details of the programming language for us and gives us essential information about instruction required to morph the code.

- Morphing:

The provided AST is then morphed with the techniques explained in 4.4. Esprima provides the AST in a JSON format and morphing the JSON would refer to changing the values at certain keys and rearranging certain key-value pairs.

- Convert AST to Code: The abstract syntax tree after morphing is converted back to JavaScript code using the module escodegen [31]. Escodegen [31] has the reverse functionality of esprima where it converts the AST back to its code. Thus, the end result of this would be a morphed code. With every iteration we can morph with different permutations of the techniques mentioned and provide with a different version of the same code everytime. 


\subsection{Architecture Overview}

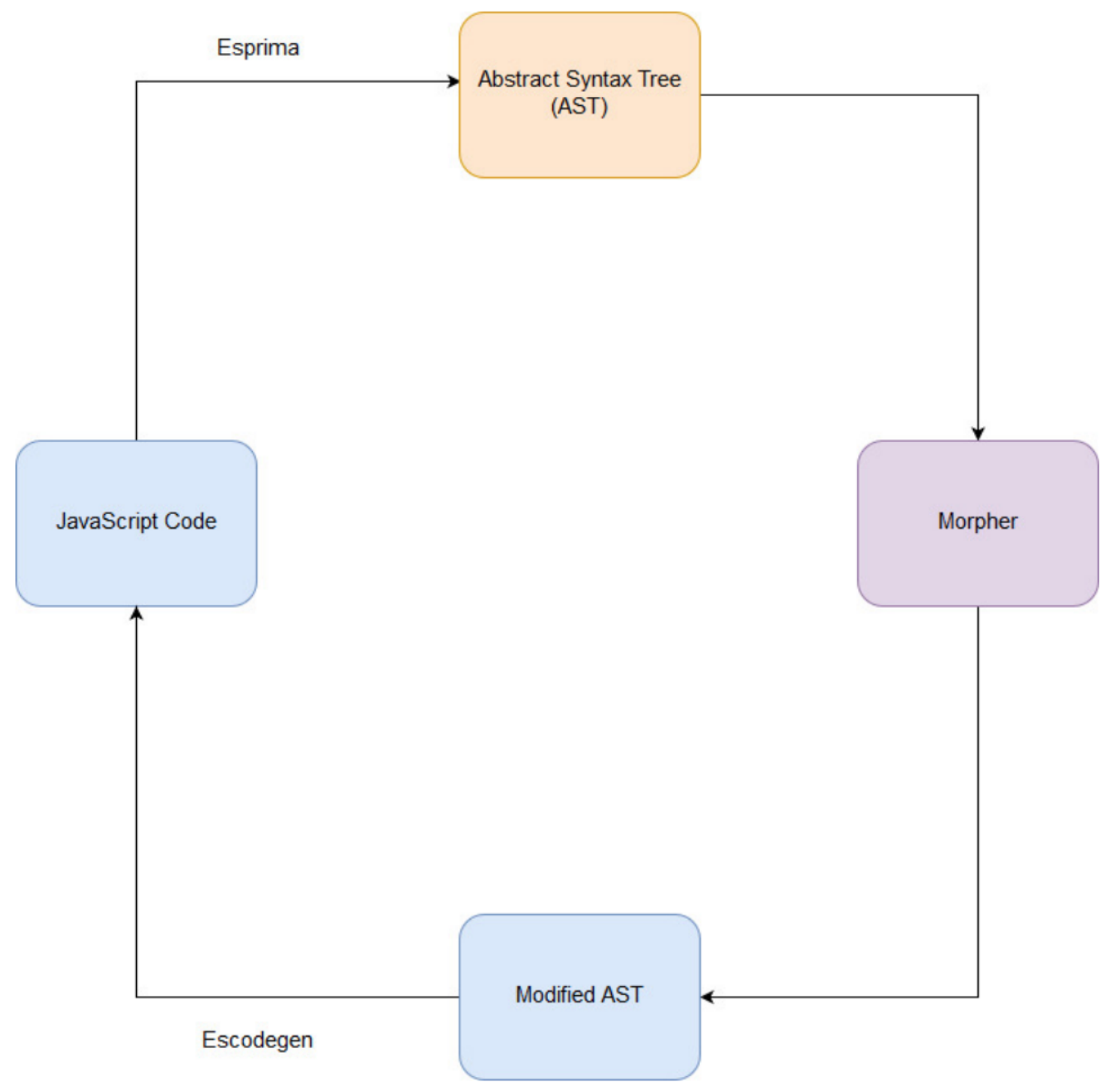

Figure 4: Morphing Engine Architecture

In the architecture, JavaScript code can be morphed to many levels. The original code is morphed using the techniques explained in 4.4 and converted back to a JavaScript code. Again, this code can be morphed further with the same set of techniques providing different levels of morphing. This will eventually make the code extremely hard for detection. Even though, with introducing such high level of morphing, the code would logically be the same. Just that, with every new generation it would have a different physical appearance. 
Listing 4.2: Original Code

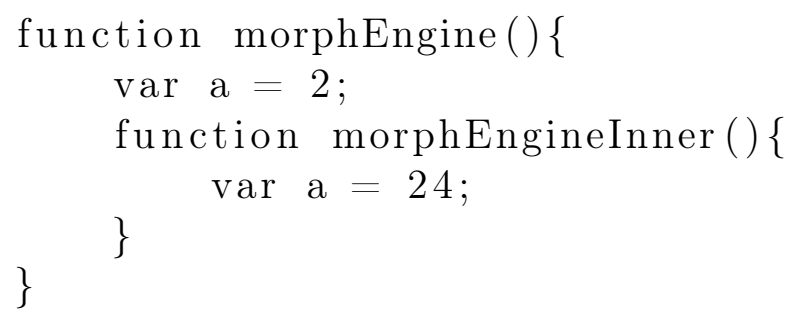

\subsection{Techniques Used}

This is the heart of the architecture, where the code is actually obfuscated. Morphing is done on the Abstract Syntax Tree provided by esprima. The following techniques are performed on the recieved AST JSON.

\subsubsection{Variable Renaming}

Variable renaming refers to changing the name of the variable in the entire code and maintaining a representation of a scope. In JavaScript we can have functions inside a function having same named variables. We need to keep track of the scope we are in to change any variable names. The data structure used is a stack. With every scope, we add the variables on the top of the stack contained in the current scope. We then change variables inside that scope using the variables on top of the stack. While parsing the AST, we only change the Variable declarations in the code. A new generated variable is assigned to respective variables for which a key-value pair is maintained.

In the Listing 4.2, the variable "a" above is declared in different functional scopes and hence will have different definitions of the variable. We need to account for both these variables. Listing 4.3 is the code after morphing. 


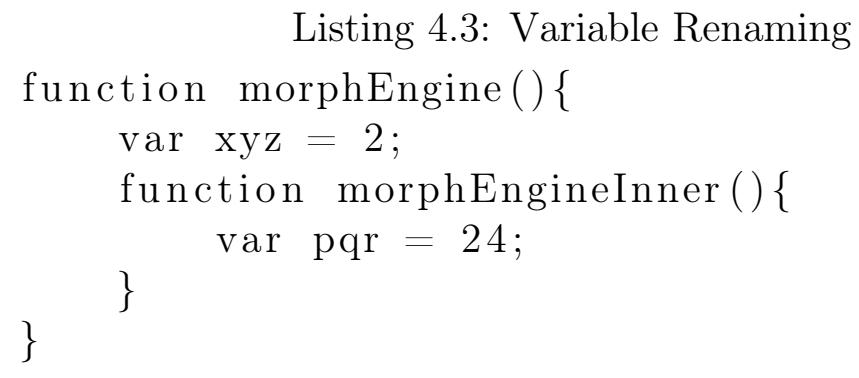

\subsubsection{Dead Code Insertion}

In this technique we insert pieces of code in the original code that changes the signature of the original code without changing its functionality. In our implementation we count the number of instructions and use that number as a seed to generate a random number, which decides the number of times the dead code will be inserted and the position of the insertion of the code is also randomized. This will ensure that there is no pattern that can be found using subsequent morphed codes. In our example, I have inserted no-operation instructions like empty function, empty console.log, empty setTimeout functions, etc. as explained in the Listing 4.4

Thus, in this we have inserted setTimeout, console.log. This does change the signature as it looks different than the original code. But these code will not include any logical changes as explained in Listing 4.5

\subsubsection{Instruction Reordering}

For this morphing technique we find out variable declaration instructions from the abstract syntax tree. We make sure that the instructions are independent and 


\section{Listing 4.5: Dead Code Insertion}

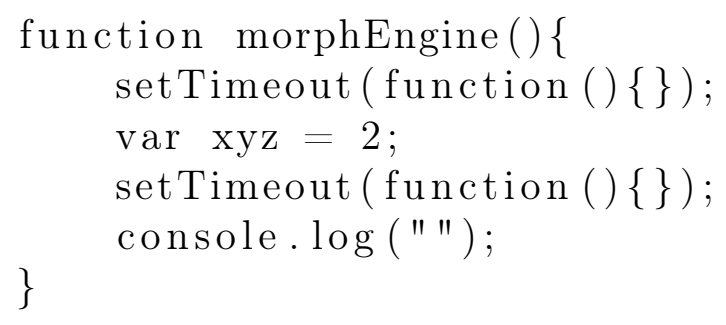

Listing 4.6: Original Code

function morphEngine()\{

var $\mathrm{a}=2$;

var $\mathrm{b}=5$;

\}

continuous to minimize the risk of introduction of bugs. Once such instructions are identified we swap them. This will swap the order of the declaration of the variable and help in changing the signature of the code as explained in Listing 4.6. After instruction reordering, we get the code in Listing 4.7. This doesn't change the logical structure of the code, but only permutes various variable declarations.

\subsubsection{Function Reordering}

Similar to instruction reordering, functions can be reordered in various permutations and combinations. Suppose, with n different functions, we can arrange it in $\mathrm{n}$ ! different ways giving $\mathrm{n}$ ! different morphed generations of the code [9] as explained in Listing 4.8. Permutations are done on this code, since there are 2 functions, it can be

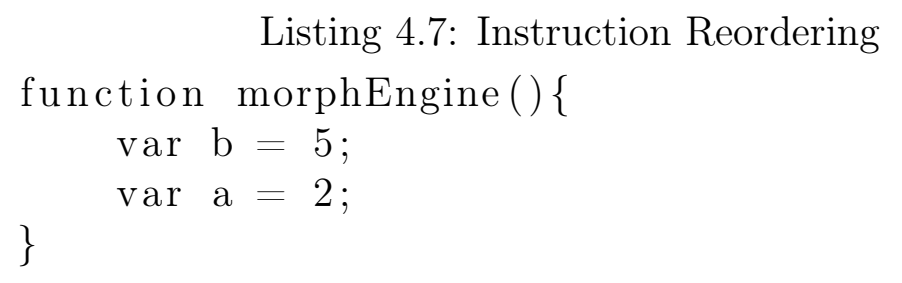

Listing 4.7: Instruction Reordering 
Listing 4.8: Original Code

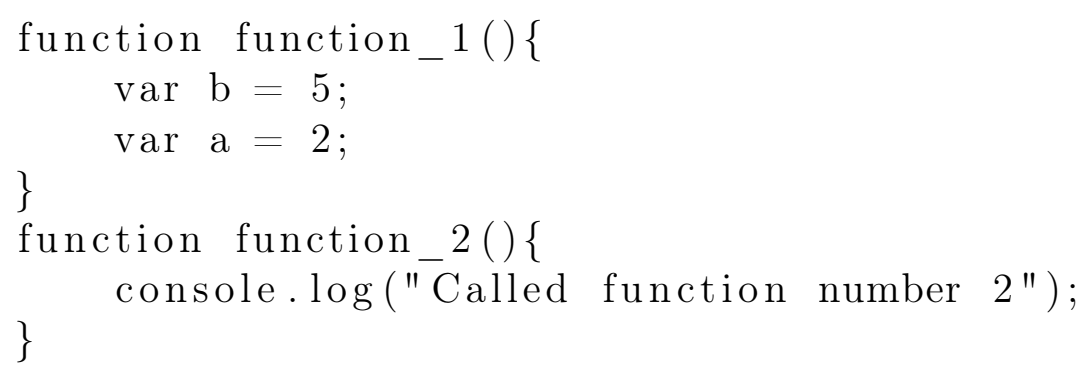

Listing 4.9: Function reordering

function function_2()\{ console.log ("Called function number 2"); \}

arranged in $2 !=2$ ways. Thus, the resulting code would look like in Listing 4.9

\subsubsection{Instruction Substitution}

Arithmetic instruction substitution is done in which the arithmetic operators are substituted with other operators. Best example is when we add 2 numbers, for example, if we have an expression var $\mathrm{a}=2+2$, it will be replaced with var $\mathrm{a}=$ $2--2$ which would result in the same result. The Original code is given in Listing 4.10 and it will be replaced by code in Listing 4.11.

With these techniques, the end result will be a completely morphed code, with completely different physical structure but will be logically the same. This morphing can be applied many times over the same code or on morphed code to provide different physical structures. 


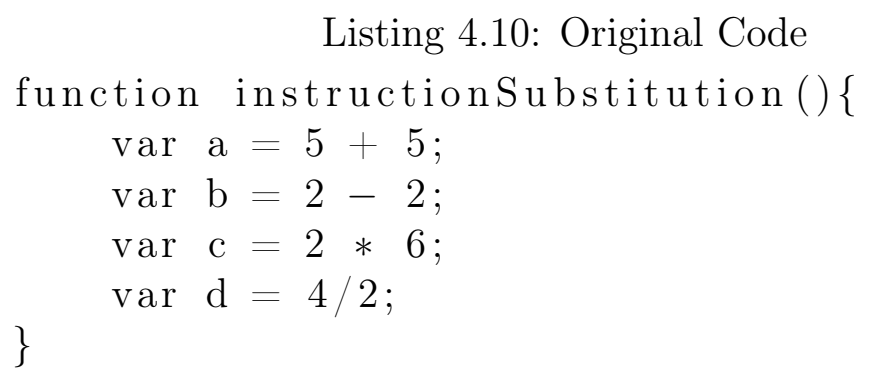

Listing 4.11: Instruction Substitution

function instructionSubstitution () \{

$$
\begin{aligned}
& \operatorname{var} \mathrm{a}=5--5 ; \\
& \operatorname{var} \mathrm{b}=2+-2 ; \\
& \operatorname{var} \mathrm{c}=2 * 12 / 2 ; \\
& \operatorname{var} \mathrm{d}=4 * 1 / 2 ;
\end{aligned}
$$




\section{CHAPTER 5}

\section{Rhino}

Netscape created a project called as Rhino which is a Javscript Engine written in Java. Rhino can be used to convert JavaScript code to Java classes and run in one of the two modes - compiled or interpreted. Javascript code is converted to Java bytecode and then run as a Java program to improve the execution time of Javascript.

\subsection{Architecture}

Rhino is based on a four step process:

- Parser

- Bytecode generator

- Interpreter

- JIT

The first phase is parsing where the code is converted into an Abstract Syntax Tree (AST). In second phase, this AST is given to a Bytecode generator, which generates a JAVA bytecode. The Rhino Interpreter then takes this bytecode as an input and converts it into a machine code with the help of JIT. This machine code is then executed.

Block diagram [3] of Rhino JavaScript can be used for reference. 


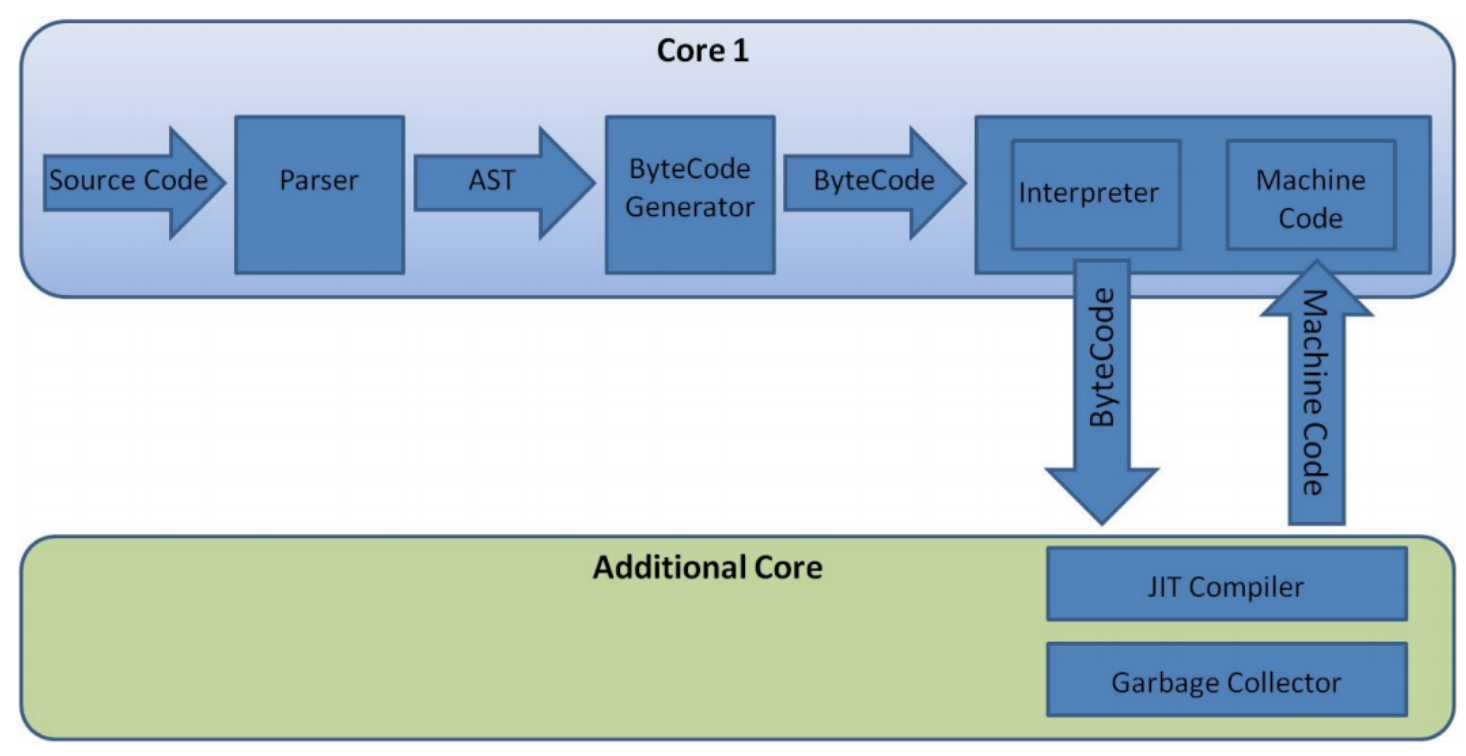

Figure 5: Rhino Block Diagram [3]

Parser: Parser has information of all the Javascript literals. It will tokenize the Javascript code and use it to generate AST. In the generated syntax tree, each leaf node represent the operands and each non-leaf node represents the operation like subtraction, comparison, addition, equivalence, etc. Greatest Common Divisor(GCD) example by binary algorithm [9] is given in Listing 5.1. The AST for the Listing 5.1 is given in Figure 6 
Listing 5.1: GCD example [9]

function GCD ( num1, num2 )

\{

$$
\begin{aligned}
& \text { if } \quad(\operatorname{num} 2>\text { num1 })\{ \\
& \quad \text { var temp = num1 } \\
& \text { num1 }=\text { num2 ; } \\
& \text { num2 }=\text { temp ; }
\end{aligned}
$$

while (true ) \{

num1 $\%=$ num2 ;

if $($ num1 $=0)$ return num2;

num2 $\%=$ num1 ;

if $(\operatorname{num} 2=0)$ return num1;

\}

\}

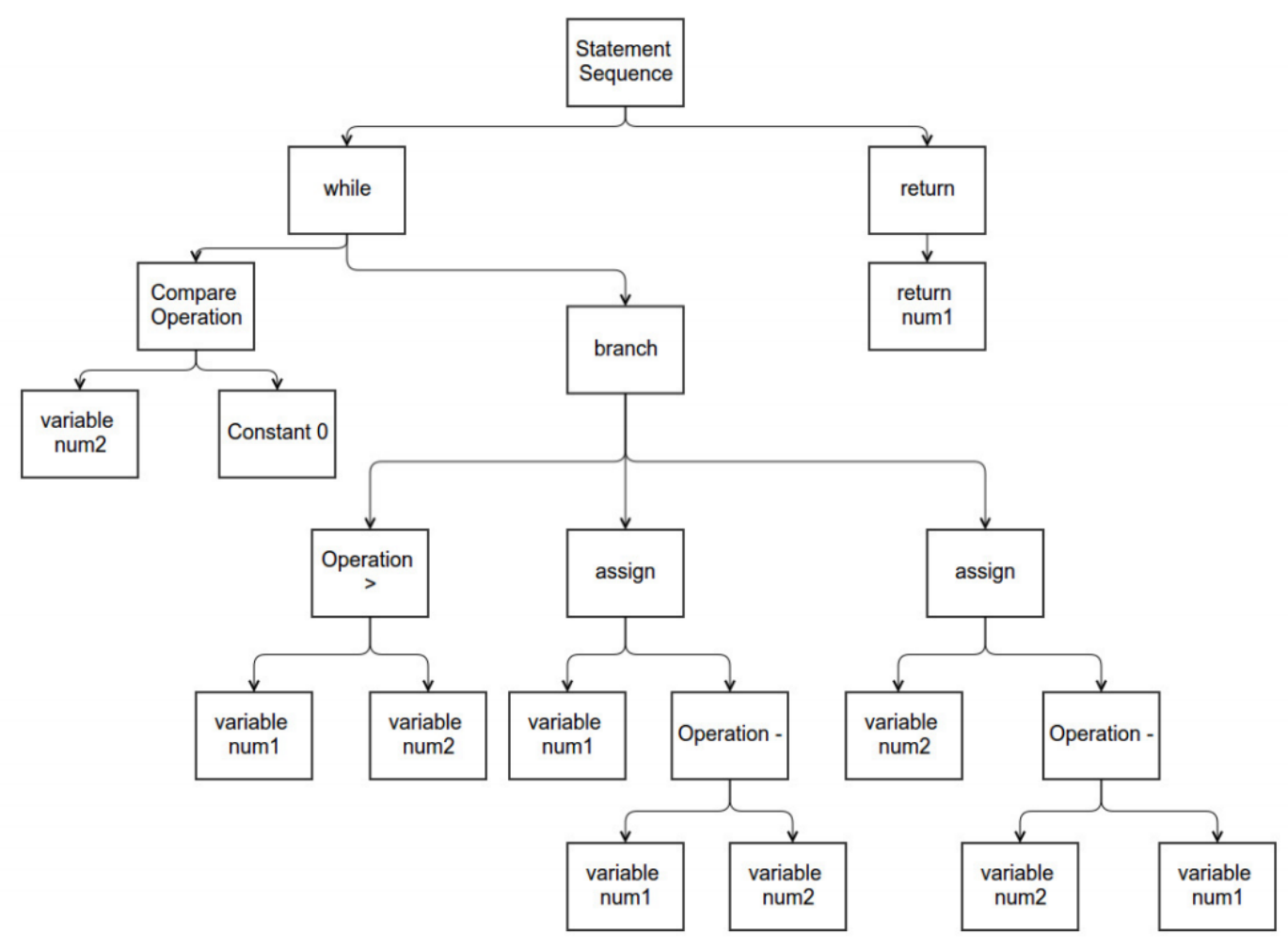

Figure 6: Abstract Syntax Tree [3] 
Bytecode Generator: AST as given as an input to the Bytecode generator. The Bytecode generator converts AST into bytecode.

For example, if there are two variables that need to be added, the generator will select that block from the AST and convert it into bytecode.

iload_1

iload_2

iadd 3

istore 3

These are explained as load first and second variable at offset 1 and 2 in the register. Add those two values and store it at offset 3 .

Interpreter: The Interpreter converts the bytecode generated by the Bytecode generator as input. This bytecode is then converted to machine level code with the help of JIT which is executed at runtime. For example, the above bytecode is given as:

MOV EAX OxFF20

MOV EBX OXFF24

SUB EAX EBX

MOV ECX EAX

\section{$5.2 \quad$ Steps}

Consider a file named as test.js. The contents of the file are:

\section{Listing 5.2: test.js}

function rhinoFile () \{

$\operatorname{var} \mathrm{a}=5+5$;

\} 
This Javascript file can be converted to Java bytecode representation using the following command:

$$
\begin{aligned}
& \text { java -cp /Users/awadhwani/Downloads/rhino1_7R4/js.jar } \\
& \text { org.mozilla.javascript.tools.jsc.Main rhinoFile.js }
\end{aligned}
$$

This gives a rhinoFile.class file which can be then used to convert to bytecode by using:

$$
\text { javap -c rhinoFile.class }
$$

The output of this command gives the following result:

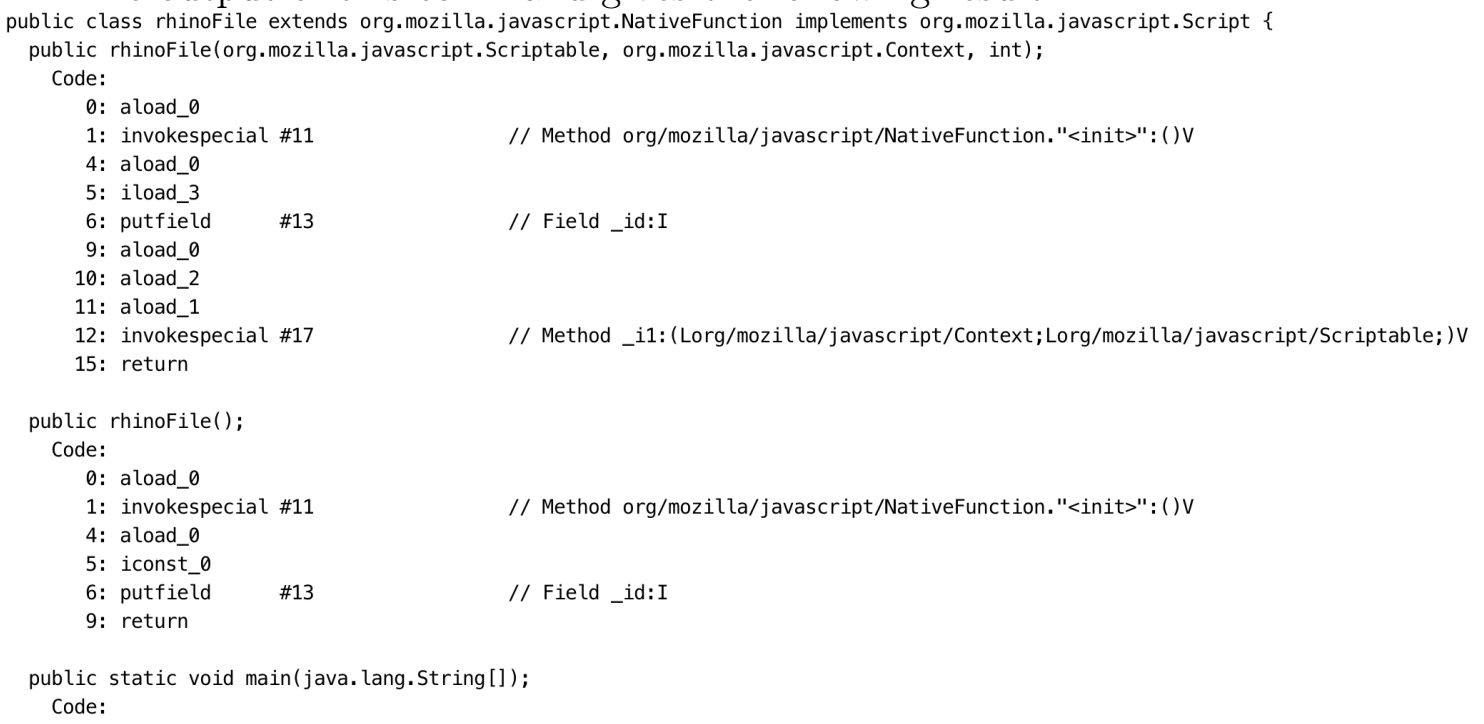

Figure 7: rhinoFile.js Bytecode 


\section{CHAPTER 6}

\section{Detecting Metamorphic JavaScript}

The primary purpose is to detect metamorphic malware from a set of given files. The purpose of metamorphism is to reduce the accuracy of the classifier to detect it as a malware. If for example a classifier clearly understands the difference between a malware and benign file, introducing metamorphism will break the model's accuracy and thus the model would misclassify malware files as benign. The aim here is to detect such malware files that are classified as benign, basically we want to detect false negatives that are introduced because of the morphing engine.

We want to classify the morphed code from the original code to be able to tell that the given file is still a malware file. Section 6.1 gives information about the dataset used. Section 6.2 explains the preprocessing required on the source code to extract features explained in section 6.3. Section 6.4 is used for training on the features extracted and running machine learning classification to classify morphed and non morphed source code.

\subsection{Dataset}

The benign dataset [32] is a set of $150 \mathrm{~K}$ benign JavaScript files. These files are collected from GitHub repositories and are filtered and processed by removing all the duplicate files, removing project forks and keeping the programs that can be parsed.

As part of the malware dataset, we use 40,000 malware samples from a github repository of JavaScript malware collection [33]. These files are parsed from github repository and it consists of JavaScript files from standard libraries and frameworks present on github. We have used entire 40,000 malware samples from the mentioned repository for malware samples and 40,000 benign samples from the benign dataset. All files from both the dataset are of the.$j$ s format.

For the purpose of having a morphed dataset, we use the morphing engine 
mentioned in Chapter 4. The malware dataset is morphed using the techniques like variable renaming, dead code insertion, function reordering, instruction substitution and instruction substitution and generated newer versions of the files which can reduce the accuracy of the classification model explained in Chapter 7. Thus, we generate 40,000 morphed samples of malware.

\subsection{Preprocessing}

The source code cannot be directly compared to the other source code to determine if the given file is harmful or not. The given input file is a.$j$ s format file and has to be preprocessed to generate features that a machine learning classifier will train on. Bytecodes are extracted from Rhino Javascript Engine explained in Chapter 5. Figure 7 shows sample bytecode file extracted from Javascript code in Section 5.2. After extraction of bytecode files, opcode sequences in those files are extracted. According to the official documentation of JAVA SE [34], a JVM instruction consist of an opcode, which specifies the operation to be performed. The opcode is followed by zero or more operands which are values that are to be operated upon. Examples of opcode are aload_0, iload_3, aload_2, tableswitch. The complete list of opcodes are specified in the official Oracle docs of JAVA SE [34]. These opcodes are extracted from the bytecode sequence for every file and placed in a sequence file where every line represents a file. Figure 8 shows the sequence file snippet.

Every line in the file is a sequence of opcodes present in the bytecode of the file. Thus, opcode sequence files are generated for benign, malware and morphed dataset. Post creation of these files, we extract the features explained in section 6.3. 
putstatic ldc2_w invokestatic putstatic sipush invokestatic putstatic ldc_w invor invokespecial putfield invokespecial invokespecial putfield new invokespecial invc tic invokestatic getfield tableswitch getfield tableswitch getfield tableswitch g $\epsilon$ irtual invokevirtual invokevirtual invokevirtual invokevirtual invokevirtual invor ual sipush invokevirtual getfield tableswitch tableswitch tableswitch invokespecial putfield tableswitch invokespecial invokespecial invokespecial putfj ield tableswitch invokestatic invokestatic invokestatic getfield tableswitch getfj switch sipush invokevirtual bipush invokevirtual bipush sipush invokevirtual getfj invokespecial putfield invokespecial invokespecial putfield new invokespecial invc tic invokestatic getfield tableswitch getfield tableswitch getfield tableswitch ge switch invokespecial putfield tableswitch invokespecial invokespecial invokespecial invor kestatic invokevirtual invokestatic ifne aload invokestatic aload getfield tablesh invokestatic getfield tableswitch getfield tableswitch getfield tableswitch bipust itch getfield tableswitch sipush invokevirtual bipush bipush invokevirtual bipush

Figure 8: Example sequence file

\subsection{Feature Extraction}

With the sequence file as input we need to extract features to be trained into a machine learning model [35]. Feature extraction is done in two ways.

1. One way is to extract n-grams [36] [26] from the sequence file, in which the value of $\mathrm{N}$ can vary. The process of extracting features from n-grams is explained in 6.3.1.

2. Second way is to extract HMM A, B and $\pi$ matrix for each file from the sequence file. This approach is explained in the section 6.5

\subsubsection{N-gram Extraction}

$\mathrm{N}$-grams are a set of words occurring together in a given window size. We move one word at each window and take the next set of words. N-grams are used in text mining and Natural language processing tasks [37]. Since N-grams are based on sequence of the words, it is the right fit in source code features [38].

Following is an example of N-grams extracted from a sequence file: For $\mathrm{N}=5$, the $\mathrm{N}$-gram features can be: 


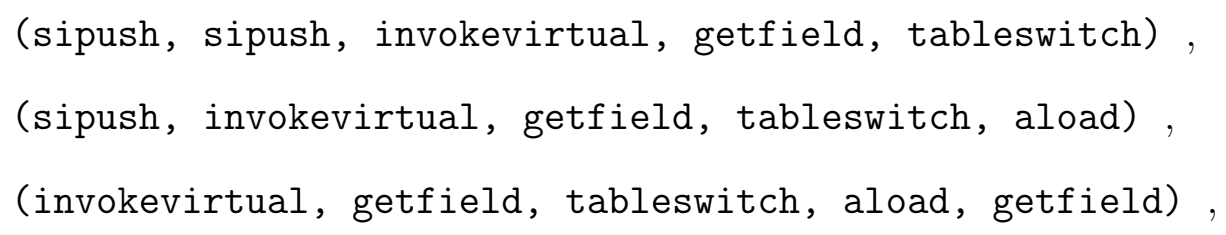

If we are planning to classify between morphed and non-morphed code. The process of extracting feature vectors would be as follows:

1. Extract N-grams from both the given sequence files (morphed and non-morphed).

2. Find out the count of all N-grams present in both files.

3. Take highest $\mathrm{K}$ occurring $\mathrm{N}$-grams as the ground truth for generating feature vectors.

4. Create vector with size $\mathrm{K}$ for every line in the individual sequence file.

5. Loop for all the lines in the file and mark the index as frequency of the n-gram on that index is present in highest $\mathrm{K}$ n-gram vector, otherwise 0 .

6. Consider the final vector array as feature vectors to be given for classification.

For example, we take top $100 \mathrm{~N}$-grams with the value of $\mathrm{N}=5$, frequency wise from the entire dataset containing morphed and non-morphed files. Each line in the sequence files is a 100 size vector with value on a index is the frequency of the N-gram in that line, otherwise 0. Similar work for malware classification is done using n-grams in [39] So, feature vector may look like: $[12,4,5,33,78,0,1, \ldots \ldots \ldots 12,3]$ - Line 1 of morphed file , $[2,55,25,36,9,0,0, \ldots \ldots \ldots, 9]$ - Line 2 of morphed file Length of feature vector $=100$ These feature vectors are then given to machine learning models for classification. 


\subsubsection{HMM feature extraction}

Another approach to extract features is by training the HMM model on an observation sequence. In this method, we take top 26 occurring opcodes from the dataset. In our example, when we take top occurring opcodes we get: ['invokestatic', 'sipush', 'putstatic', 'ldc_w', 'invokespecial', ..] These opcodes are then represented in numbers starting from 0 .

So, invokestatic $=0$, sipush $=1$, putstatic $=2$ and so on. Thus, nonmorphed and morphed sequence files are replaced by numeric mappings of the opcodes given in the map formed. We only take top 26 occurring opcodes, rest every other opcode is taken as other $=26$ Thus, eventually an observation sequence file would look like:

$[1,5,18,3,3,0,11,7, \ldots]$

This observation sequence is extracted for both types of sequence files and taken as input for HMM models.

\subsection{Training using N-gram}

Post feature extraction, we train the machine learning models explained in the below sections. These models take N-gram generated feature vectors and train on them. Cross-validation with $80-20$ split is applied.

\subsubsection{SVM}

SVMs is a supervised learning model. SVM algorithm finds a hyperplane in a N-dimensional space that would separate points of different classes [40]. Data points can come on either side of the hyperplane and the objective is to maximize the distance between data points and the hyperplane. Thus, by separating the points we can have achive classification between the data points. It is well suited for malware detection as explained in [20]. 


\subsubsection{Random Forest}

Random forest is a supervised learning algorithm. In Random Forest, we have many decision trees working in parallel. A decision tree is a machine learning model in which decisions are taken at attributes and a tree like structure is created which eventually holds a class. The basic structure of Random Forest is when we merge many decision trees [40]. The idea behind this is that we can combine decision tree models to increase the overall accuracy of the model.

\subsubsection{K Nearest Neighbors}

In k-nearest neighbor, a class of a point in Euclidean space is determined by calculating distance between every other point [40]. Majority of the top k classes distance wise are taken for the point in question. In voting process, whatever majority of the classes are for the $\mathrm{k}$ points, that class is assigned to that point. Value of $\mathrm{k}$ is taken to be odd, since there should never be a tie.

\subsubsection{Naive Bayes}

Naive Bayes works on definition the probability of features in all the classes with having classes from c_0 through c_k and features from $\mathrm{x}_{-} 0$ through $\mathrm{x} \_\mathrm{n}$. Using this, it will determine the class of the point in question. For this, we use the Bayes rule [41].

$$
P(\mathbf{A} \mid \mathbf{B})=P(\mathbf{A}) \frac{P(\mathbf{B} \mid \mathbf{A})}{P(\mathbf{B})}
$$

\subsection{HMM}

As explained in section 6.3.2, we extract observation sequence for morphed as well as non morphed dataset. Using these observation sequences, multiple experiments are run on it. We change the value of $\mathrm{N}$ with every experiment and observe the results. 


\subsubsection{Training}

The training phase of HMM is explained as Problem 3 in section 2.3.4. Using the dataset explained in section 6.1 for both malware non-morphed and malware morphed, we strip out $\mathrm{T}=50,000$ opcodes and train the HMM with 1000 iterations. The value of $\mathrm{M}$ is taken as 26 since we have 26 top occurring opcode symbols and $\mathrm{N}$ is taken as $[2,3,5]$ for experimentation. A, B and $\pi$ matrix are initialized randomly making them row stochastic.

The HMM follows the estimate and maximize(EM) algorithm [42]. In the maximize step the observation vector is aligned with the state in that model and the log likelihood is maximized. The estimate step estimates the parameters for vectors that are aligned to the state and the state transition probabilities. There is a maximize and estimate step for each iteration. This repeats for all iterations mentioned in the model.

\subsubsection{Classification using HMM vectors}

As explained in the section 6.4, N-gram vectors are used to train the machine learning models like SVM, Random Forest, K Nearest Neighbors, Feed forward Neural Network. Though these provide good accuracy in terms of detection as mentioned in the 7 section. We could have different set of feature vectors which are explained in section 6.3.2.

Each HMM run gives a A, B and $\pi$ matrix. These values could be merged together and used as feature vectors. So for each $\mathrm{HMM}$ with $\mathrm{N}=2$, we get a $2 \times 2$ A matrix and a $2 \times 26 \mathrm{~B}$ matrix. If we merge these two together we get a $2 \times 28$ matrix which can be then flattened and used as a feature vector. Thus, our feature vector here would be a $1 \times 56$ vector for each HMM run. As the HMM is run for both types of sequence files - morphed and non morphed, we get feature vectors for both the classes. 
For example, consider A matrix as

$$
\left[\begin{array}{ll}
a_{11} & a_{12} \\
a_{21} & a_{22}
\end{array}\right]
$$

Consider B matrix as

$$
\left[\begin{array}{lllll}
b_{11} & b_{12} & b_{13} & \ldots & b_{126} \\
b_{21} & b_{22} & b_{23} & \ldots & b_{226}
\end{array}\right]
$$

When we merge these two and flatten we get,

$$
\left[\begin{array}{llllllllllll}
a_{11} & a_{12} & b_{11} & b_{12} & \ldots & b_{126} & a_{21} & a_{22} & b_{21} & b_{22} & \ldots & b_{226}
\end{array}\right]
$$

This matrix is then used as a feature vector for one HMMs that run on 50,000 opcodes from one type of sequence file. The process is explained in section 6.5. Non morphed sequence file HMM feature vectors are given a class 0, representing non-morph class and Morphed sequence file HMM feature vectors are given a class 1, representing morph class. After this, it is a standard machine learning classification problem. After we receive these feature vectors, we can use all the classification models explained in 6.4. It is observed that these features give better accuracy as compared to N-gram features as explained in 7 . 


\section{CHAPTER 7}

\section{Experiments}

This chapter concentrates on the experiments carried out and results of those experiments covered in 7.2. It also explains the setup used for these experiments in section 7.1 .

The focus of the experiments is two-fold:

1. First part concentrates on reducing the accuracy of a malware detector by inducing code morphing.

2. The second part concentrates on increasing the accuracy of morph code detector.

\subsection{Setup}

A virtual machine is used for training malware samples. Oracle VirtualBox is used for maintaining a virtual machine. Table 2 explains the host machine specifications and Table 3 explains the guest machine specifications.

Feature extraction for N-grams and HMM feature vectors is done using the guest machine as we deal with malware files. After we extract the feature vectors, we predict the results on the host machine. The classification based on machine learning models and prediction is done on the host machine.

\begin{tabular}{||cc||}
\hline Attribute & Specification \\
\hline \hline Model & MacBook Pro (Retina, 15-inch, Mid 2015) \\
\hline Processor & $2.5 \mathrm{GHz}$ Intel Core i7 \\
\hline Memory & 16 GB 1600 MHz DDR3 \\
\hline System Type & 64 -bit OS \\
\hline Operating System & macOs Mojave 10.14.3 \\
\hline
\end{tabular}

Table 2: Host Machine Specifications 


\begin{tabular}{||cc||}
\hline Attribute & Specification \\
\hline \hline Software & Oracle VirtualBox Version 6.0 .4 r128413 \\
\hline Base memory & $4096 \mathrm{MB}$ \\
\hline Processors & 4 \\
\hline System Type & 64-bit OS \\
\hline Operating System & Linux Ubuntu 12.04.3 LTS \\
\hline
\end{tabular}

Table 3: Guest Machine Specifications

\subsection{Results}

The first section focuses on reducing the accuracy of existing malware detectors. The next section focuses on building a morphed code detector using N-gram frequency and HMM vector as features.

\subsubsection{Reducing the accuracy of malware detector}

The primary purpose of this section is to explain how code morphing can reduce the accuracy of an existing malware detector. These techniques are explained in Chapter 4. The techniques implemented are:

- Inserting No-operation instruction.

- Variable Renaming

- Instruction Reordering

- Function Reordering

The malware detector is trained on malware and benign dataset explained in section 6.1. Various machine learning models are used for the classification.

- K Nearest Neighbours

- Random Forest

- Support Vector Machine

- Naive Bayes

These models with varied parameters are trained with malware and benign dataset. 
Accuracy is taken as a measure of comparison. Classifcation models are judged by plotting Receiver Operating Characteristics curve [43]. Area under the curve explains the correctness of classification models.

a) Random Forest

Random Forest achieves the AUC-ROC of 0.93 with 100 trees in the forest.

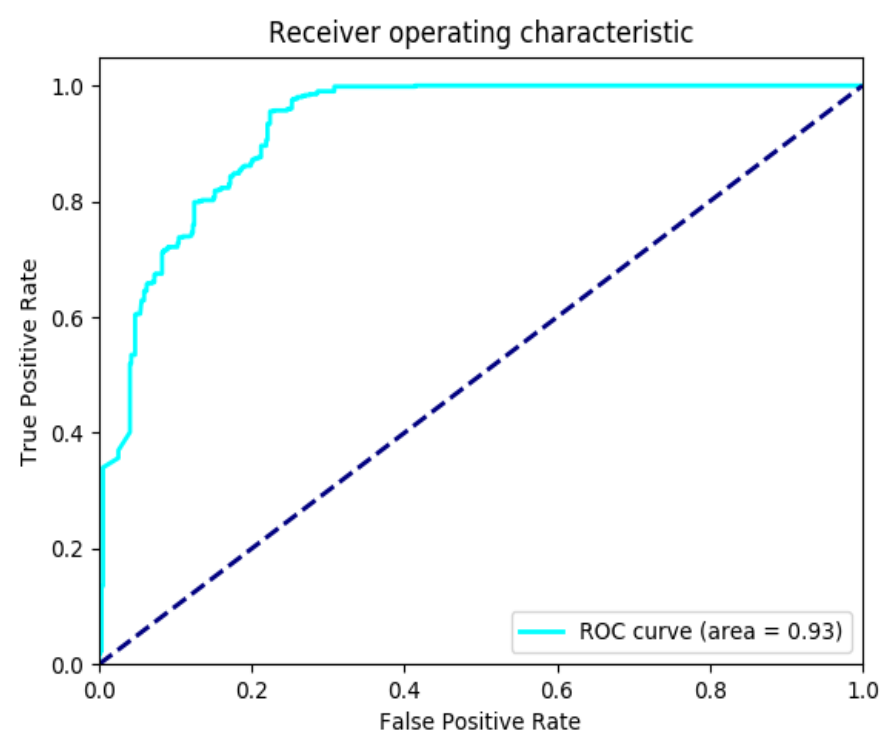

Figure 9: Random Forest 
b) K Nearest Neighbours

K Nearest Neighbors achieves AUC-ROC of 0.94 . This is with value of $\mathrm{K}=5$

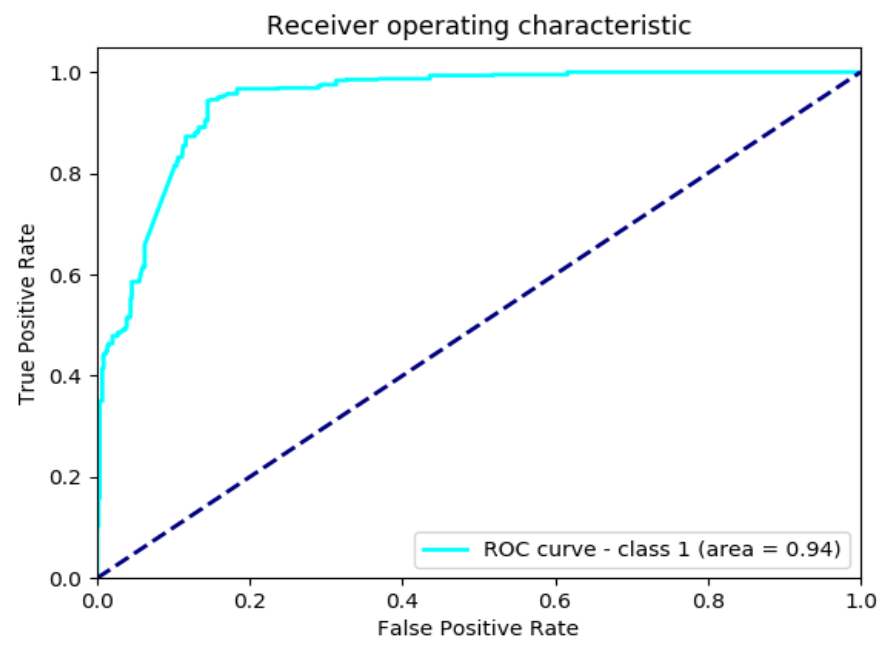

Figure 10: K Nearest Neighbours

c) Support Vector Machine

Support Vector Machine achieves AUC-ROC of 0.96. Kernel used is linear

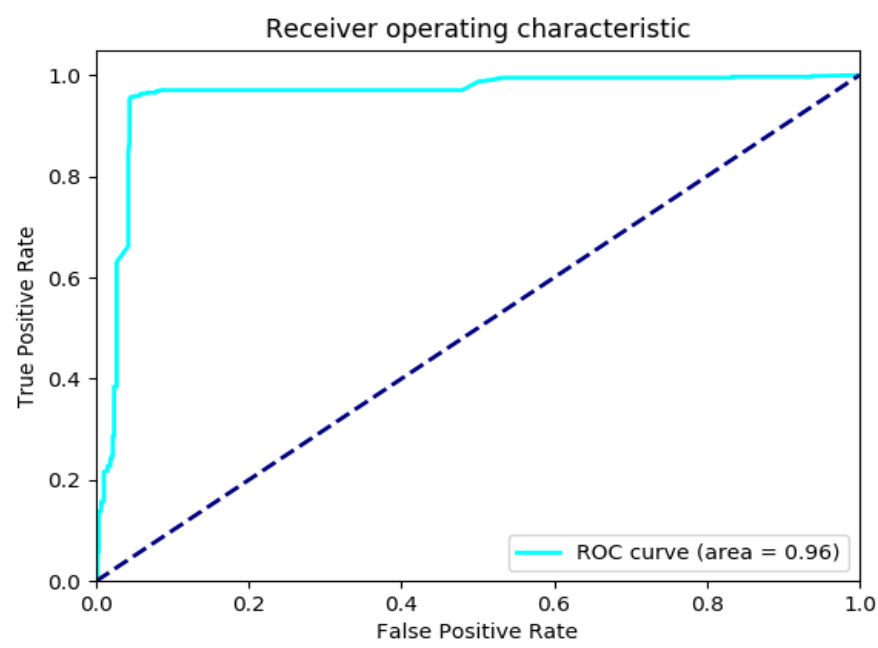

Figure 11: Support Vector Machine 
d) Naïve Bayes - AUC-ROC - 0.94

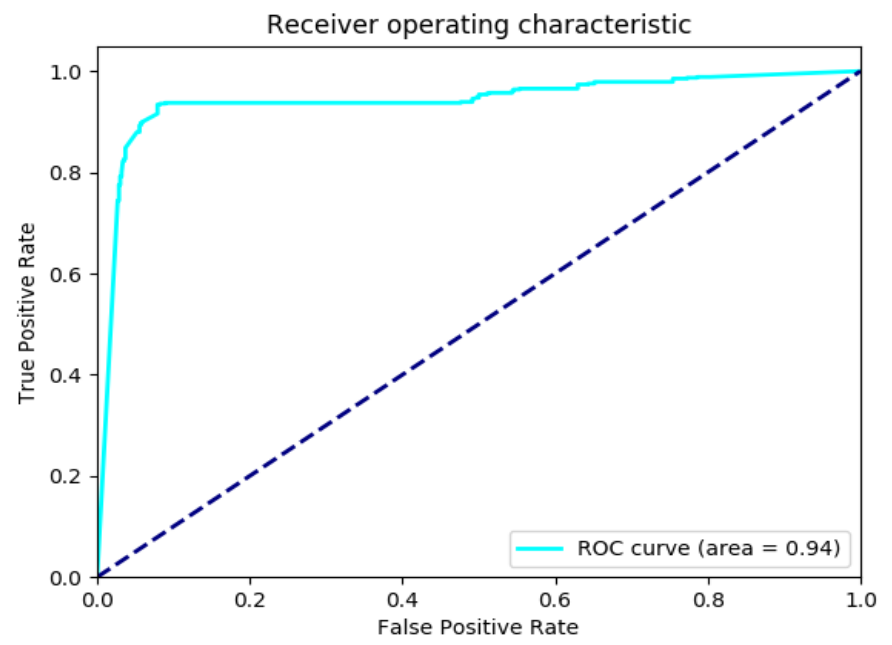

Figure 12: Naïve Bayes

In the results above, SVM with linear kernel gives the best results of malware detection with accuracy of $95.25 \%$. For SVM, post application of the morphing techniques, the accuracy of the model is degraded to $73.3 \%$. Table 4 depict the degraded accuracy's of various models. These results are obtained with one level of morphing. K-Nearest Neighbor has the max accuracy drop of $37.3 \%$.

\section{Table 4: Accuracy Drop}

\begin{tabular}{||cccc||}
\hline Model & Accuracy & Morphed Accuracy & Accuracy Drop \\
\hline \hline KNN & $93.5 \%$ & $56.2 \%$ & $37.3 \%$ \\
\hline Random Forest & $86.5 \%$ & $54.55 \%$ & $31.95 \%$ \\
\hline SVM & $95.25 \%$ & $73.3 \%$ & $21.95 \%$ \\
\hline Naïve Bayes & $85.75 \%$ & $49 \%$ & $36.75 \%$ \\
\hline
\end{tabular}




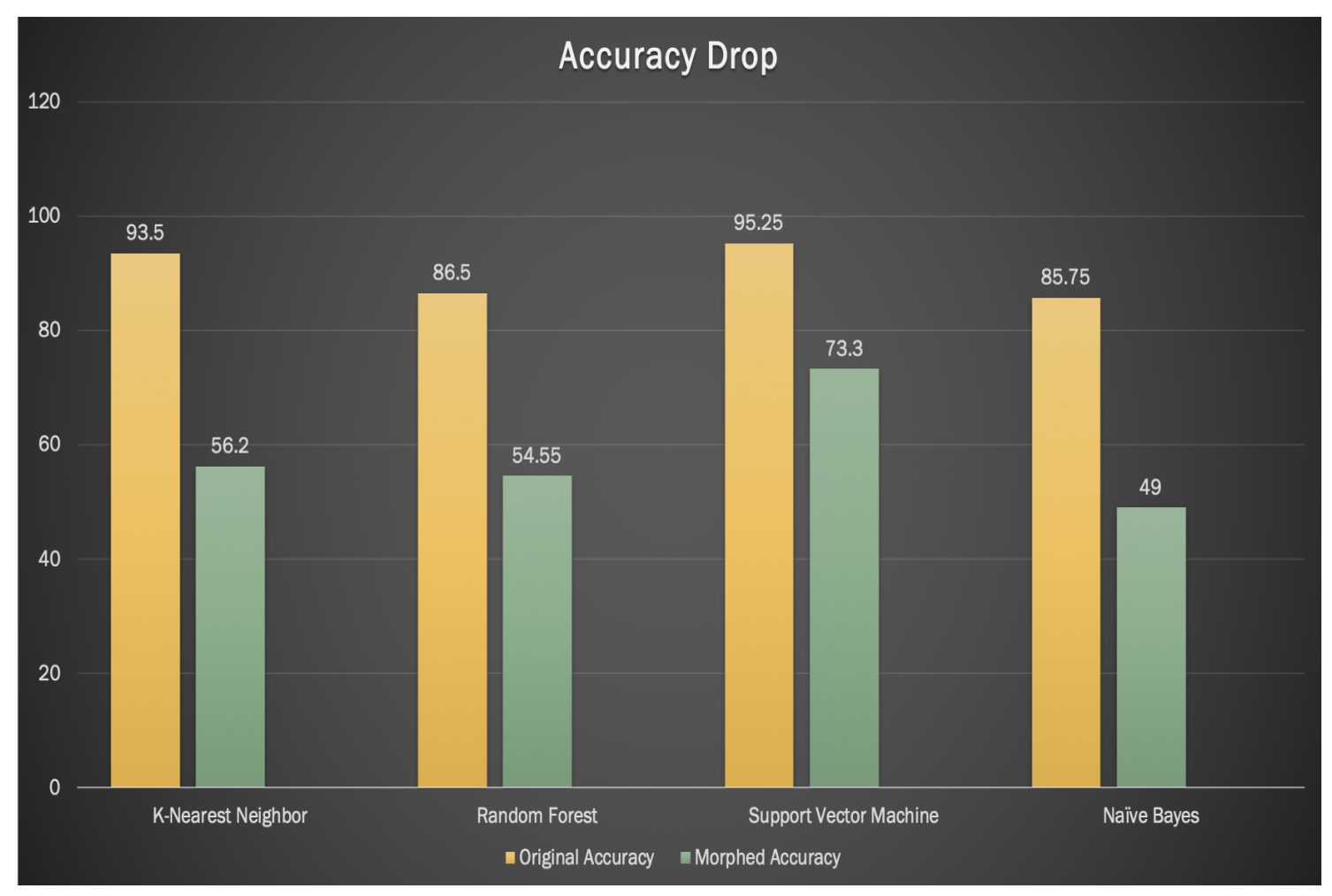

Figure 13: Accuracy Drop Graph

Figure 13 shows the accuracy drop, the $\mathrm{x}$-axis represents the four classifiers: Random Forest, Naïve Bayes, K Nearest Neighbors, and Support Vector Machine. The y-axis shows the accuracy for all the classifiers. The yellow bars show the original accuracy and the green bars show the morphed accuracy after applying morphing techniques explained in Section 4. Figure 14 shows the effect of dead code on accuracy. On the $\mathrm{x}$-axis we have percentage of dead code added in the files, on y-axis we have accuracy. So, as and when we increase the amount of dead code, the accuracy keeps decreasing. For $75 \%$ of dead code, accuracy drops till $47 \%$.

Thus, classifiers accuracy can be dropped significantly using code morphing. The next section concentrates on training the model to learn morphing using the same machine learning models and also using HMM. As explained in the section 2.3.4, HMM is a statistical model well suited for sequential analysis. Thus, experiments are done 
using HMM and are explained in the next section.

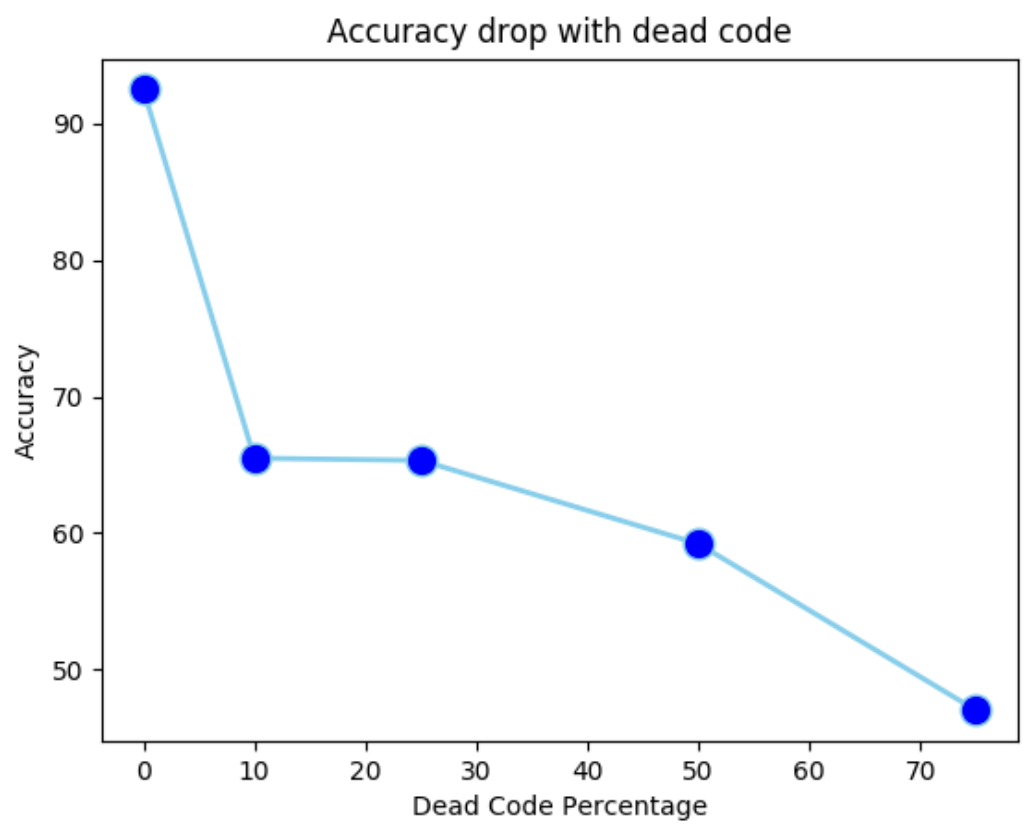

Figure 14: Accuracy Drop - Dead Code

\subsubsection{Building a metamorphic detector}

Morphing malware have reduced the significance of signature based detection as it becomes very difficult to know each variant of the morphed code [44]. Thus, it is very important to experiment on techniques that could detect metamorphic malware. The experiments in this section involve using machine learning models explained in chapter 6 .

We will first experiment by using feature vectors explained in section 6.3.1. These features are created from the idea of the existence of top n-grams available in a file. The idea is that if a malware file consists of a given set of common n-grams, and if the file in question has a majority of them, we classify that file as malware. Even after morphing techniques like introducing new code, changing variables would not change the entire flow of opcodes. 
a) Random Forest using $\mathrm{N}$-grams with $\mathrm{N}=5$

Random Forest achieves the AUC-ROC of 0.97 with 100 trees in the forest.

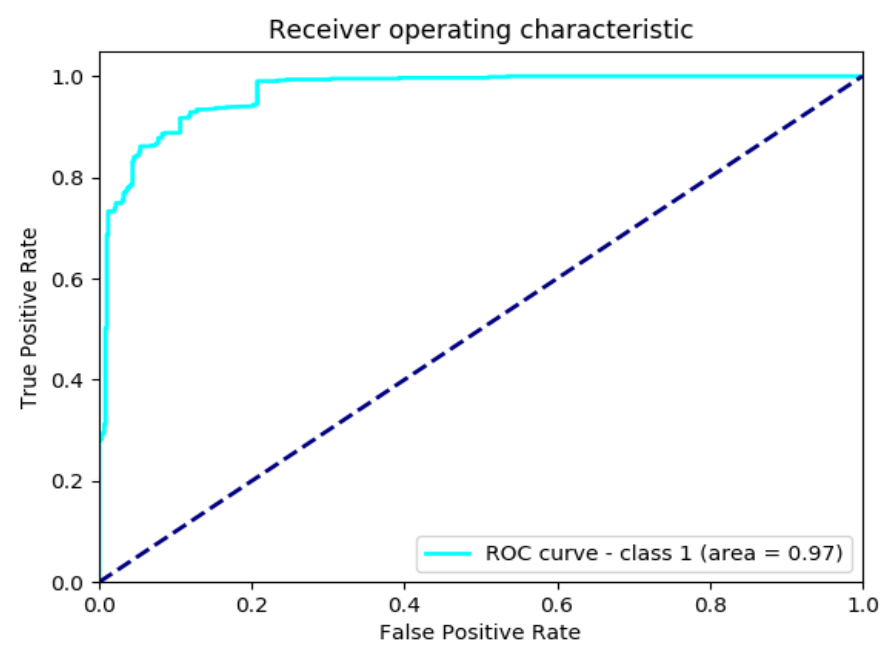

Figure 15: Random Forest using N-grams

b) K-Nearest Neighbours using $\mathrm{N}$-grams with $\mathrm{N}=5, \mathrm{~K}=5$

K Nearest Neighbors achieves AUC-ROC of 0.99. This is with value of $K=5$

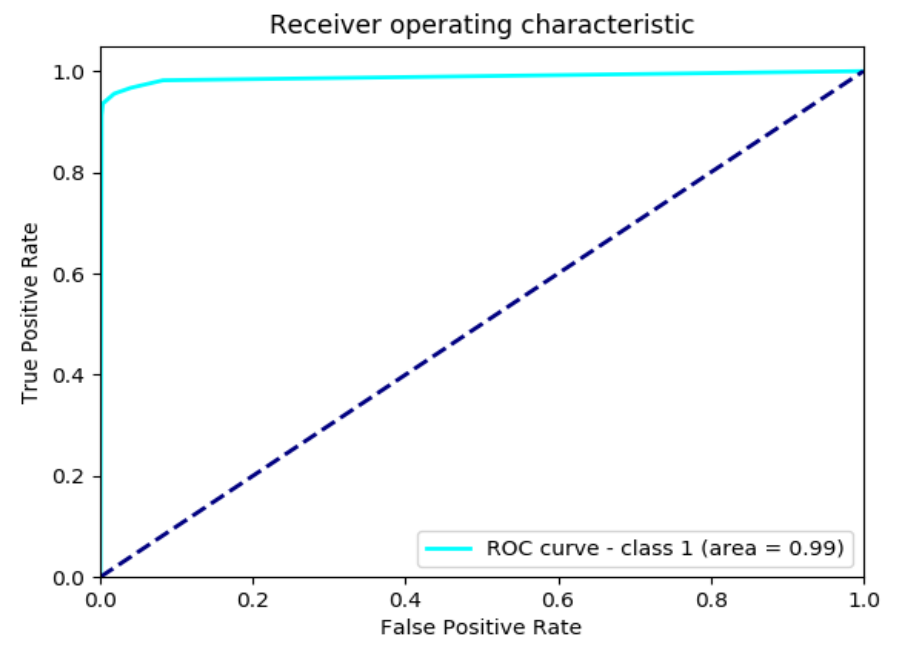

Figure 16: K Nearest Neighbours using N-grams 
c) Support Vector Machine using $\mathrm{N}$-grams with $\mathrm{N}=5$

Support Vector Machine achieves AUC-ROC of 0.98. Kernel used is 'linear'

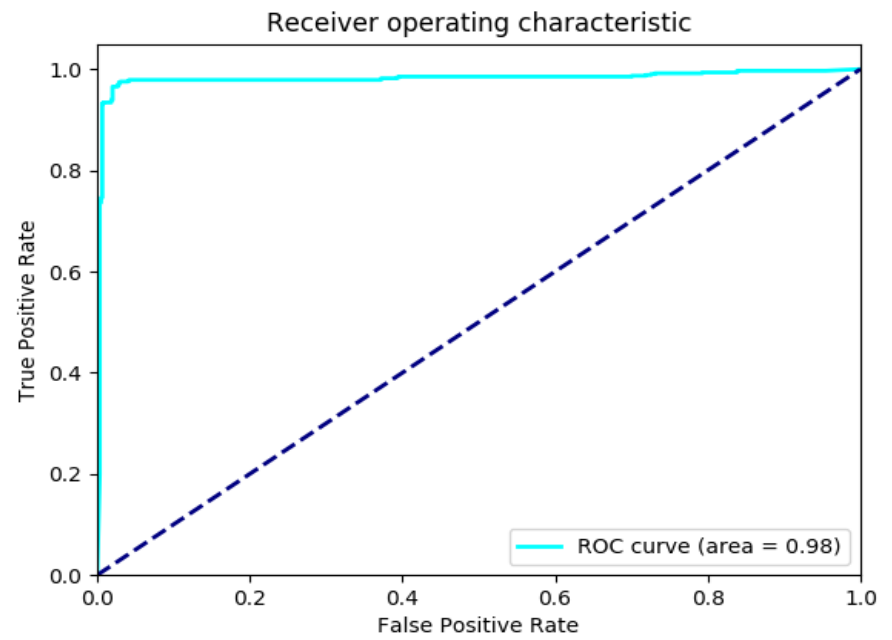

Figure 17: Support Vector Machine using N-grams

d) Naive Bayes using N-grams with $\mathrm{N}=5$ - AUC-ROC - 0.81

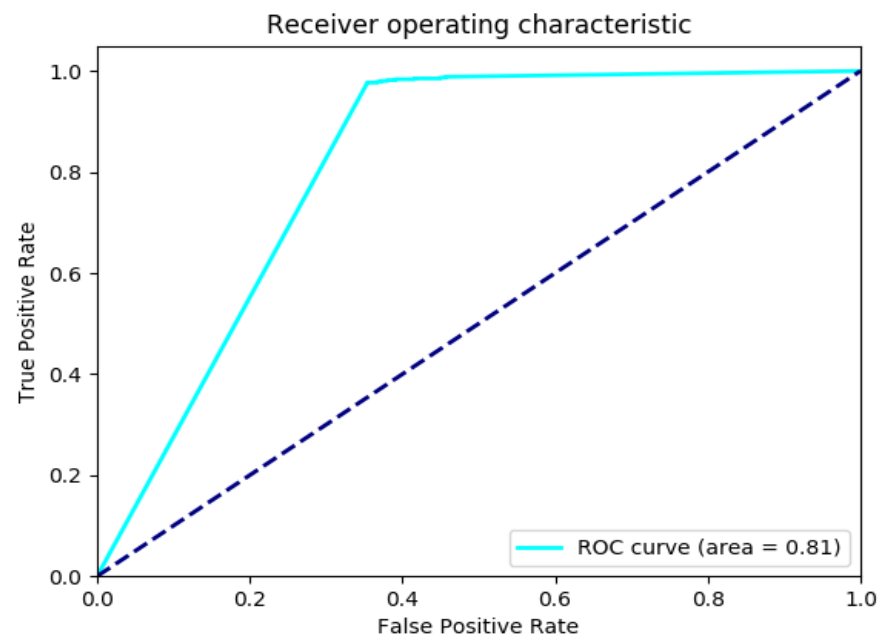

Figure 18: Naive Bayes using N-grams

We experiment with different values of $\mathrm{k}$ in KNN. The values of $\mathrm{K}$ used are 3,5,7 and 11. In case of Random Forest we use 100 trees. The number of trees are also varied and 100, 200 and 300 are used for training. 
The second approach is to use HMM to train on morphed sequences. We use two HMM models in which one we train on a malware opcode sequence from 40,000 files and the other one we train on morphed malware opcode sequences from 40,000 morphed samples. The HMM specifications used are $\mathrm{N}=[2,3,5], \mathrm{M}=26$, iterations $=1000$ and random row stochastic values for A, B and $\pi$ matrix. The HMM is run on $\mathrm{T}=50,000$ opcode sequences from the two sequence files. The two HMM models provide final A, B and $\pi$ matrix for both type of sequence files. Thus, as explained in section 6.3 .2 , we merge the $\mathrm{A}, \mathrm{B}$ and $\pi$ matrix to get a feature vector and we can run classification on them.

After we receive feature vectors, it becomes a machine learning classification problem. Thus we can apply standard machine learning techniques to classify on the basis of the feature vectors. The following experiments are performed with $\mathrm{N}=2$. Experiments with varied values of $\mathrm{N}$ is presented in Appendix $\mathrm{A}$

a) Random Forest using HMM vectors with $\mathrm{N}=2$

Random Forest achieves the AUC-ROC of 0.99 with 100 trees in the forest.

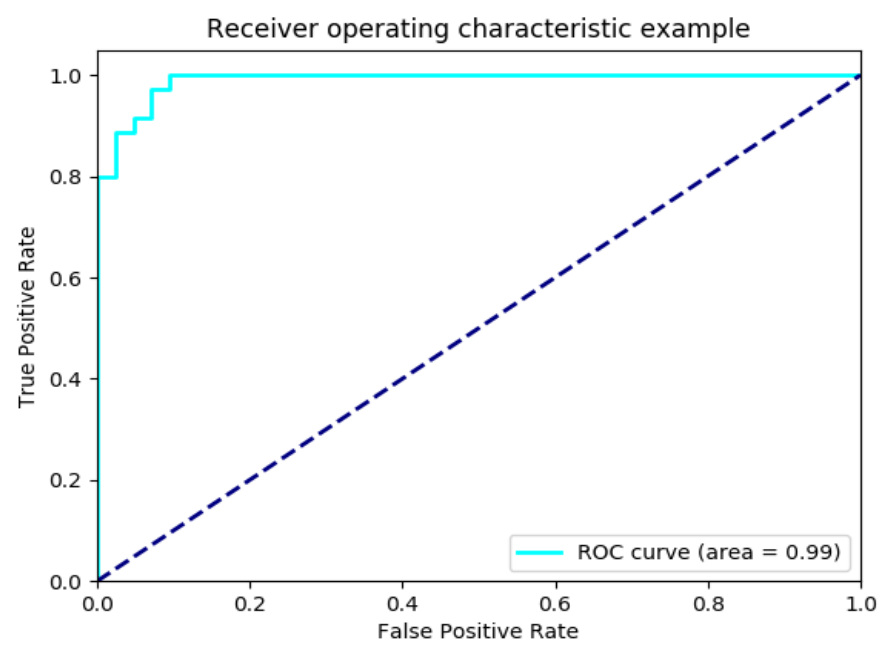

Figure 19: Random Forest using HMM vectors 
b) K Nearest Neighbours using HMM vectors with $\mathrm{N}=2$

K Nearest Neighbors achieves AUC-ROC of 0.86 . This is with value of $\mathrm{K}=5$

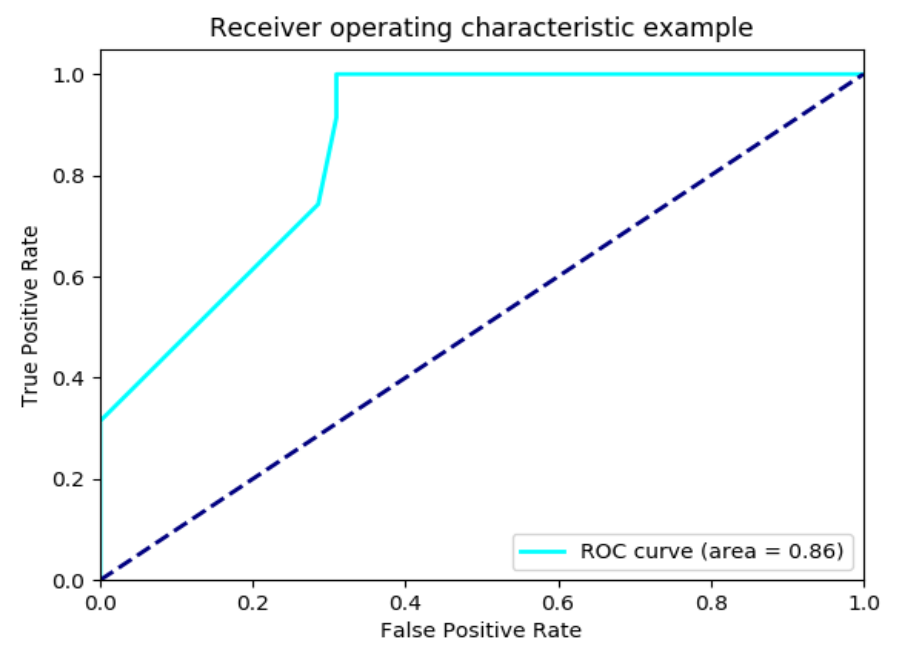

Figure 20: K Nearest Neighbours using HMM vectors

c) Support Vector Machine using HMM vectors with $\mathrm{N}=2$

Support Vector Machine achieves AUC-ROC of 0.97. Kernel used is 'linear'

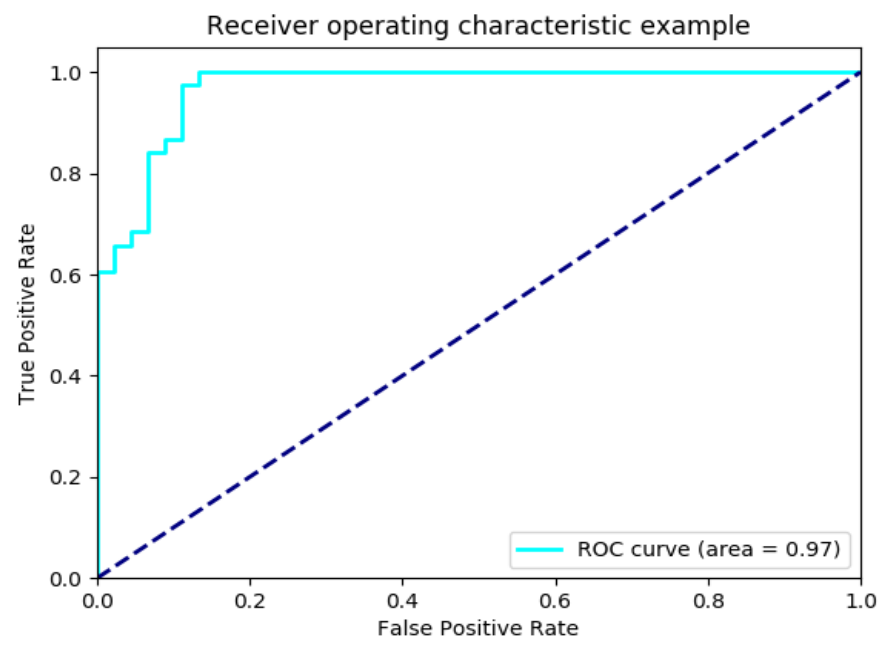

Figure 21: Support Vector Machine using HMM vectors 
d) Naive Bayes using HMM vectors with $\mathrm{N}=2$

Naive Bayes performs the worst with AUC-ROC as 0.88

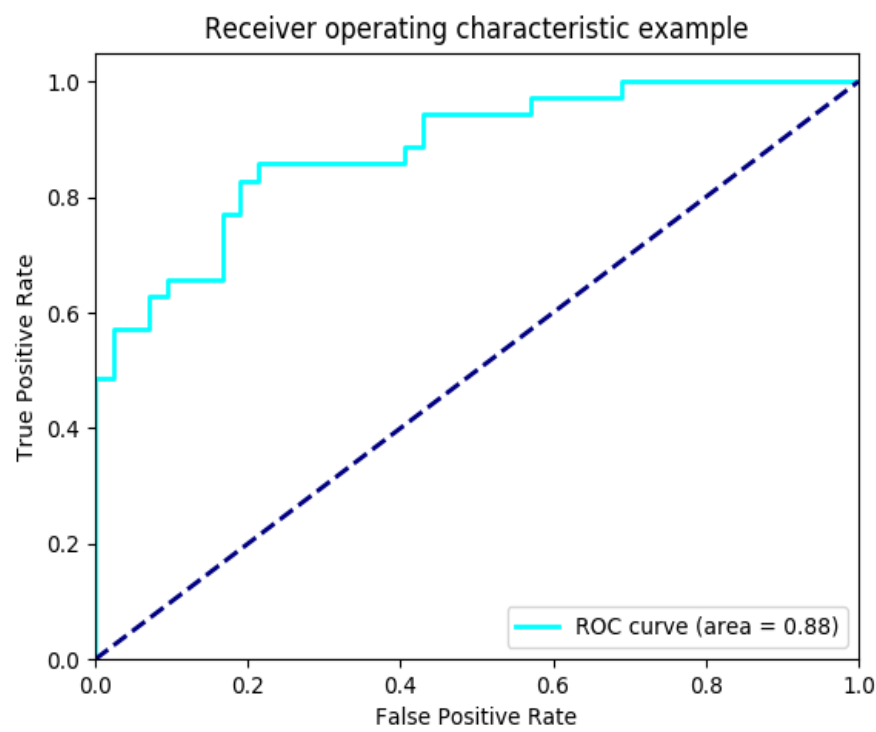

Figure 22: Naive Bayes using HMM vectors 


\section{CHAPTER 8}

\section{Conclusion and Future Work}

In this project, the aim was to detect metamorphic malware. We first introduced the metamorphic engine to morph the JavaScript based code. The techniques used are variable renaming, function reordering, instruction reordering, dead code insertion and instruction substitution. These techniques when used together provide great levels of code obfuscation. As discussed in the project, major anti-virus software programs use signature based detection for detecting malwares which would eventually fail because of code obfuscation. We are using NodeJS esprima and escodegen to convert the code to and from Abstract Syntax Tree of that code. Abstract Syntax Trees are used to morph the code by changing the logical structure using the techniques mentioned above. With this we achieve a $21.95 \%$ drop from $95.25 \%$ to $73.3 \%$ when using SVM as a malware detector with max accuracy drop of $37.3 \%$ while using K-Nearest Neighbor.

The next part of the project is to detect metamorphic code. Since we have the source code, we cannot directly compare the source files to classify malware files. Thus, we converted it into meaningful feature vectors. First approach was to use N-gram feature vectors on bytecode files of those JavaScript source codes. We use Rhino JavaScript engine to convert JavaScript code to JAVA bytecodes. The bytecode file is then used to extract opcodes from. The sequence of opcode symbols are important in the experiment rather than the entire source file. We remove opcodes from all the files and place it in a sequence file where every line of opcodes represents a file. Thus for detecting metamorphic malware, we have sequence files for malware as well as morphed malware. We then take out $\mathrm{N}$-gram frequency features and apply machine learning models like SVM, Random Forest, Naive Bayes and K Nearest Neighbor. From this, Support Vector Machine gives the best accuracy of $97 \%$ and AUC-ROC of 
0.98 .

We also experimented using HMM feature vectors. In the same sequence files for malware and morphed malware, instead of the opcodes, we represent the opcodes in numeric format. We calculate the frequency of the opcodes in the sequence files and keep only top 26 opcode symbols. Using $\mathrm{N}=[2,3,5], \mathrm{M}=26, \mathrm{~T}=50,000$ and iterations $=1000$ we train the HMM model and extract $\mathrm{A}, \mathrm{B}$ and $\pi$ matrix for each HMM output for 50,000 opcodes. These matrices are merged together and form a feature vector for classification models. After we get the feature vectors of $1 \times 56$ dimension. We use it for classification with the same machine learning models used for N-gram feature vectors. Using HMM feature vectors, SVM performs the best with 96.8\% accuracy with KNN being on the second position with $96.38 \%$ accuracy.

The main purpose of these approaches is that if a malware file is morphed, it can act as benign in most of the malware detectors. Thus, to catch such type of malware we have a metamorphic detector which would determine that there is some morphing involved in the file and needs to be scrutinized again. Thus, we mark such files as a potential candidate of being a malware. As part of future work, we can combine this approach along with dynamic analysis where we run a sandbox to understand the behavior of the files and detect malware files easily. As we followed a two-fold process by degrading the accuracy of a detector thereby increasing accuracy for morph code detection, we could experiment in the field of Malware GAN [45] where there are two neural networks that compete with each other to improve metamorphic malware creation. One neural network aims to create newer versions of malware to beat the other network which tries to differentiate between two classes. It will be an interesting field of research. We could also try to detect Transcriptase [46] malware. 


\section{LIST OF REFERENCES}

[1] M. Stamp, "A revealing introduction to Hidden Markov Models," Department of Computer Science San Jose State University, pp. 26--56, 2004.

[2] M. Statistics, "Trends report| av-test," 2017. [Online]. Available: https://www.av-test.org/fileadmin/pdf/security_report/AV-TEST_ Security_Report_2017-2018.pdf

[3] A. Prabhu, "Working of rhino." [Online]. Available: http://www.quora.com/ JavaScript-programming-language/How-does-a-JavaScript-engine-work

[4] "Greg zemskov." [Online]. Available: https://www.plesk.com/blog/producttechnology/hidden-website-threats-deal-with-site-malware

[5] Wikipedia contributors, "Sony bmg copy protection rootkit scandal --Wikipedia, the free encyclopedia," 2019, [Online; accessed 18-April-2019]. [Online]. Available: https://en.wikipedia.org/w/index.php?title=Sony_BMG_ copy_protection_rootkit_scandal\&oldid $=881854109$

[6] "Panda security (n.d.). virus, worms, trojans and backdoors: Other harmful relatives of viruses." [Online]. Available: http://www.pandasecurity.com/ homeusers-cms3/security-info/about-malware/generalconcep

[7] C. Annachhatre, T. H. Austin, and M. Stamp, "Hidden markov models for malware classification," Journal of Computer Virology and Hacking Techniques, vol. 11, no. 2, pp. 59--73, 2015.

[8] J. Aycock, Computer Viruses and Malware. Springer US, 2006.

[9] M. Musale, T. H. Austin, and M. Stamp, "Hunting for metamorphic javascript malware," Journal of Computer Virology and Hacking Techniques, vol. 11, no. 2, pp. 89--102, 2015.

[10] X. Li, P. K. Loh, and F. Tan, "Mechanisms of polymorphic and metamorphic viruses," in 2011 European intelligence and security informatics conference. IEEE, 2011, pp. 149--154.

[11] B. B. Rad, M. Masrom, and S. Ibrahim, "Camouflage in malware: from encryption to metamorphism," International Journal of Computer Science and Network Security, vol. 12, no. 8, pp. 74--83, 2012. 
[12] A. S. Bist, "Detection of metamorphic viruses: A survey," in 2014 International Conference on Advances in Computing, Communications and Informatics (ICACCI). IEEE, 2014, pp. 1559--1565.

[13] I. You and K. Yim, "Malware obfuscation techniques: A brief survey," in 2010 International conference on broadband, wireless computing, communication and applications. IEEE, 2010, pp. 297--300.

[14] V. S. Koganti, L. K. Galla, and N. Nuthalapati, "Internet worms and its detection," in 2016 International Conference on Control, Instrumentation, Communication and Computational Technologies (ICCICCT). IEEE, 2016, pp. 64--73.

[15] Z. Bazrafshan, H. Hashemi, S. M. H. Fard, and A. Hamzeh, "A survey on heuristic malware detection techniques," in The 5th Conference on Information and Knowledge Technology. IEEE, 2013, pp. 113--120.

[16] M. Stamp, Information security: principles and practice. John Wiley \& Sons, 2011.

[17] W. Liu, P. Ren, K. Liu, and H.-x. Duan, "Behavior-based malware analysis and detection," in 2011 First International Workshop on Complexity and Data Mining. IEEE, 2011, pp. 39--42.

[18] G. Hăjmăşan, A. Mondoc, and O. Creţ, "Dynamic behavior evaluation for malware detection," in 2017 5th International Symposium on Digital Forensic and Security (ISDFS). IEEE, 2017, pp. 1--6.

[19] S. Priyadarshi, "Metamorphic detection via emulation," 2011.

[20] I. Firdausi, A. Erwin, A. S. Nugroho, et al., "Analysis of machine learning techniques used in behavior-based malware detection," in 2010 second international conference on advances in computing, control, and telecommunication technologies. IEEE, 2010, pp. 201--203.

[21] W.-J. Li, K. Wang, S. J. Stolfo, and B. Herzog, "Fileprints: Identifying file types by n-gram analysis," in Proceedings from the Sixth Annual IEEE SMC Information Assurance Workshop. IEEE, 2005, pp. 64--71.

[22] T. H. Austin, E. Filiol, S. Josse, and M. Stamp, "Exploring hidden markov models for virus analysis: a semantic approach," in 2013 46th Hawaii International Conference on System Sciences. IEEE, 2013, pp. 5039--5048.

[23] A. Krogh, "An introduction to hidden markov models for biological sequences." 
[24] M. G. Schultz, E. Eskin, F. Zadok, and S. J. Stolfo, "Data mining methods for detection of new malicious executables," in Proceedings 2001 IEEE Symposium on Security and Privacy. SESP 2001. IEEE, 2001, pp. 38--49.

[25] F. Rezaei, M. K. Nezhad, S. Rezaei, and A. Payandeh, "Detecting encrypted metamorphic viruses by hidden markov models," in 2014 11th International Conference on Fuzzy Systems and Knowledge Discovery (FSKD). IEEE, 2014, pp. 973--977.

[26] S. M. Sridhara and M. Stamp, "Metamorphic worm that carries its own morphing engine," Journal of Computer Virology and Hacking Techniques, vol. 9, no. 2, pp. 49--58, 2013.

[27] G. Blanc, D. Miyamoto, M. Akiyama, and Y. Kadobayashi, "Characterizing obfuscated javascript using abstract syntax trees: Experimenting with malicious scripts," in 2012 26th International Conference on Advanced Information Networking and Applications Workshops. IEEE, 2012, pp. 344--351.

[28] Wikipedia contributors, "Abstract syntax tree --- Wikipedia, the free encyclopedia," 2019, [Online; accessed 18-April-2019]. [Online]. Available: https:// en.wikipedia.org/w/index.php?title=Abstract_syntax_tree\&oldid=891491683

[29] J. Picado, "Abstract syntax trees on javascript." [Online]. Available: https:// medium.com/@jotadeveloper/abstract-syntax-trees-on-javascript-534e33361fc7

[30] A. Hidayat, "Esprima npm." [Online]. Available: https://www.npmjs.com/ package/esprima

[31] Y. Suzuki, "Escodegen npm." [Online]. Available: https://www.npmjs.com/ package/escodegen

[32] V. Raychev, P. Bielik, M. Vechev, and A. Krause, "Learning programs from noisy data," in ACM SIGPLAN Notices, vol. 51, no. 1. ACM, 2016, pp. 761--774.

[33] H. Petrak, "github repo: javascript-malware-collection." [Online]. Available: https://github.com/HynekPetrak/javascript-malware-collection

[34] Oracle, "Jvm instruction set." [Online]. Available: https://docs.oracle.com/ javase/specs/jvms/se7/html/jvms-6.html

[35] S. Ranveer and S. Hiray, "Comparative analysis of feature extraction methods of malware detection," International Journal of Computer Applications, vol. 120, no. 5,2015 .

[36] J. Z. Kolter and M. A. Maloof, "Learning to detect and classify malicious executables in the wild," Journal of Machine Learning Research, vol. 7, no. Dec, pp. 2721--2744, 2006. 
[37] I. Santos, Y. K. Penya, J. Devesa, and P. G. Bringas, "N-grams-based file signatures for malware detection." ICEIS (2), vol. 9, pp. 317--320, 2009.

[38] S. Jain and Y. K. Meena, "Byte level n--gram analysis for malware detection," in International Conference on Information Processing. Springer, 2011, pp. 51--59.

[39] Z. Fuyong and Z. Tiezhu, "Malware detection and classification based on n-grams attribute similarity," in 2017 IEEE International Conference on Computational Science and Engineering (CSE) and IEEE International Conference on Embedded and Ubiquitous Computing (EUC), vol. 1. IEEE, 2017, pp. 793--796.

[40] A. Singh, N. Thakur, and A. Sharma, "A review of supervised machine learning algorithms," in 2016 3rd International Conference on Computing for Sustainable Global Development (INDIACom). IEEE, 2016, pp. 1310--1315.

[41] H. Zhang and D. Li, "Naïve bayes text classifier," in 2007 IEEE International Conference on Granular Computing (GRC 2007). IEEE, 2007, pp. 708--708.

[42] J. M. (https://stats.stackexchange.com/users/44603/jeffm), "Training a hidden markov model, multiple training instances," Cross Validated, uRL:https://stats.stackexchange.com/q/95553 (version: 2014-04-29). [Online]. Available: https://stats.stackexchange.com/q/95553

[43] A. P. Bradley, "The use of the area under the roc curve in the evaluation of machine learning algorithms," Pattern recognition, vol. 30, no. 7, pp. 1145--1159, 1997.

[44] S. Choudhary and M. D. Vidyarthi, "A simple method for detection of metamorphic malware using dynamic analysis and text mining," Procedia Computer Science, vol. 54, pp. 265--270, 2015.

[45] W. Hu and Y. Tan, "Generating adversarial malware examples for black-box attacks based on gan," arXiv preprint arXiv:1702.05983, 2017.

[46] F. Di Troia, C. A. Visaggio, T. H. Austin, and M. Stamp, "Advanced transcriptase for javascript malware," in 2016 11th International Conference on Malicious and Unwanted Software (MALWARE). IEEE, 2016, pp. 1--8. 


\section{APPENDIX}

\section{Appendix A}

A.1 HMM for detecting metamorphic malware with $\mathrm{N}=3, \mathrm{M}=\mathbf{2 6}$, $\mathrm{T}=50,000$

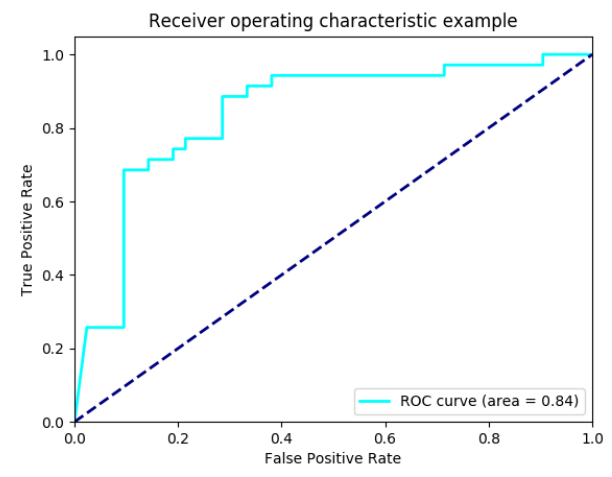

(a)

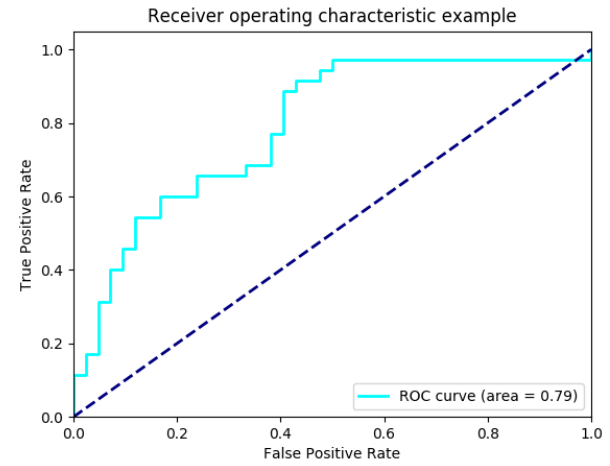

(c)

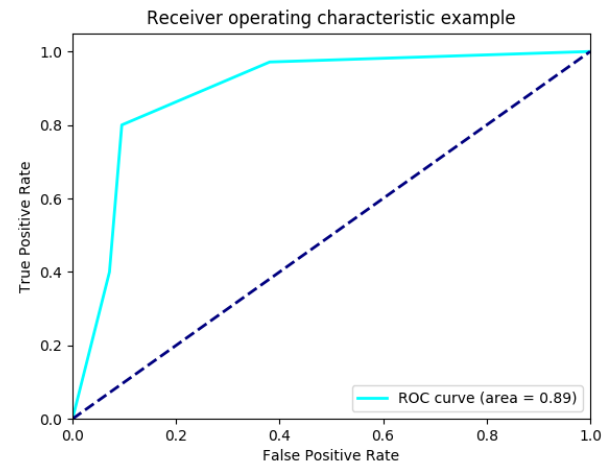

(b)

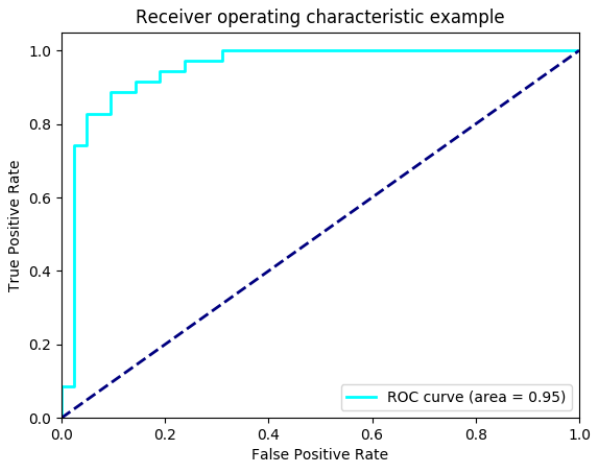

(d)

Figure A.23: HMM with $\mathrm{N}=3, \mathrm{M}=26, \mathrm{~T}=50,000$ (a) Naïve Bayes; (b) K-Nearest Neighbor; (c) Support Vector Machine; (d) Random Forest 


\section{A.2 HMM for detecting metamorphic malware with $\mathrm{N}=5, \mathrm{M}=\mathbf{2 6}$, $\mathrm{T}=50,000$}

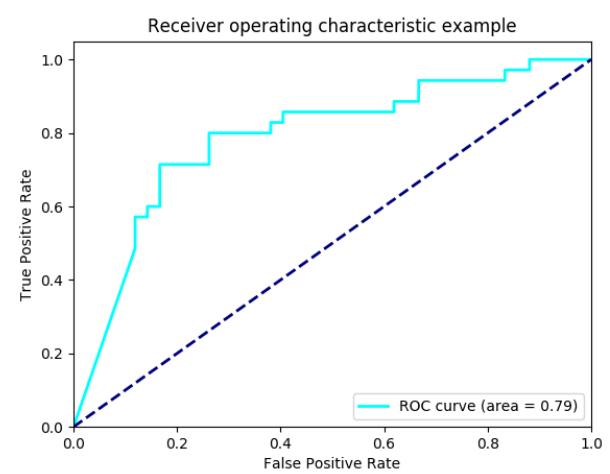

(a)

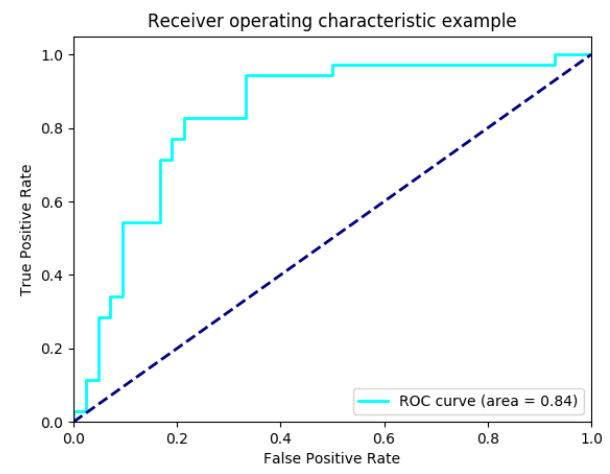

(c)

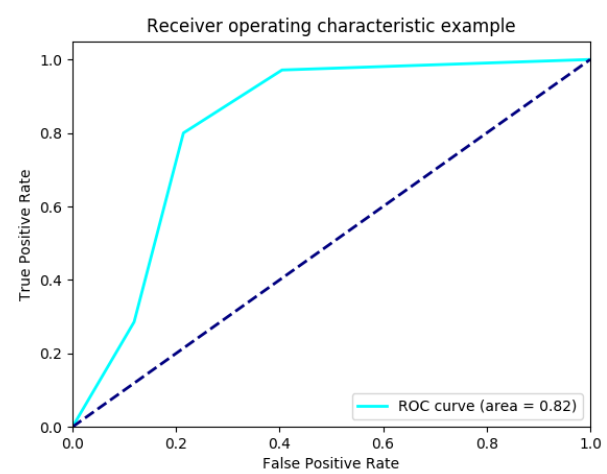

(b)

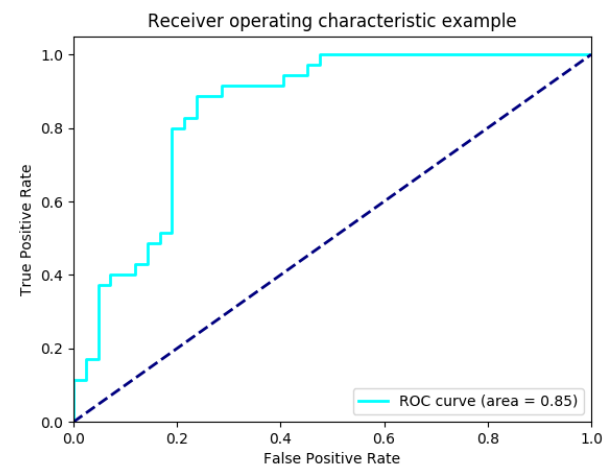

(d)

Figure A.24: HMM with $\mathrm{N}=5, \mathrm{M}=26, \mathrm{~T}=50,000$ (a) Naïve Bayes; (b) K-Nearest Neighbor; (c) Support Vector Machine; (d) Random Forest 


\section{A.3 K-Nearest Neighbors for metamorphic malware detection using N- gram features with $\mathrm{K}=[3,7,11]$}

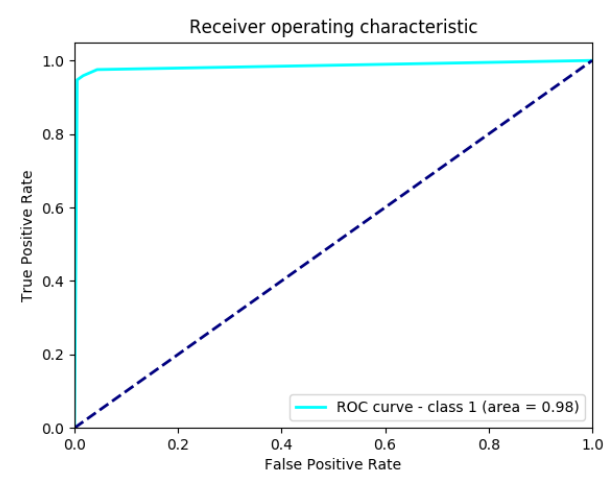

(a)

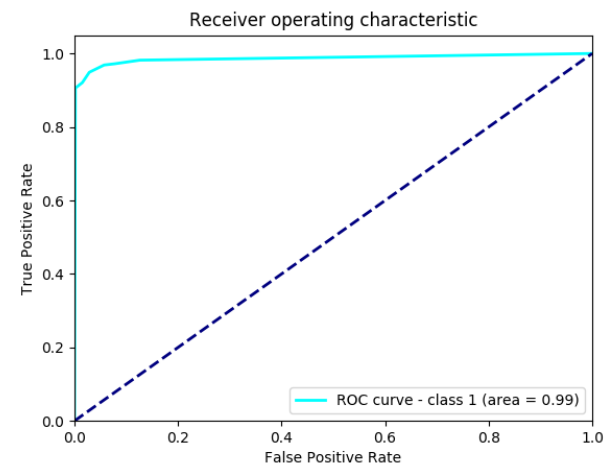

(b)

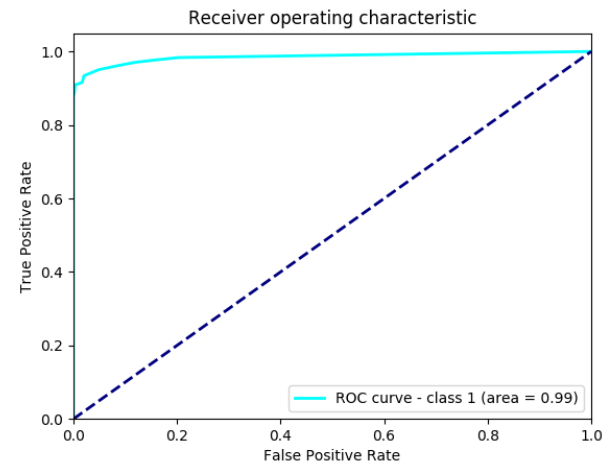

(c)

Figure A.25: N-grams with K-Nearest Neighbor (KNN) as classifier (a) KNN with $\mathrm{N}=3$; (b) KNN with $\mathrm{N}=7$; (c) $\mathrm{KNN}$ with $\mathrm{N}=11$; 


\section{A.4 Random Forest for metamorphic malware detection using N-gram features with Trees $=[200,300]$}

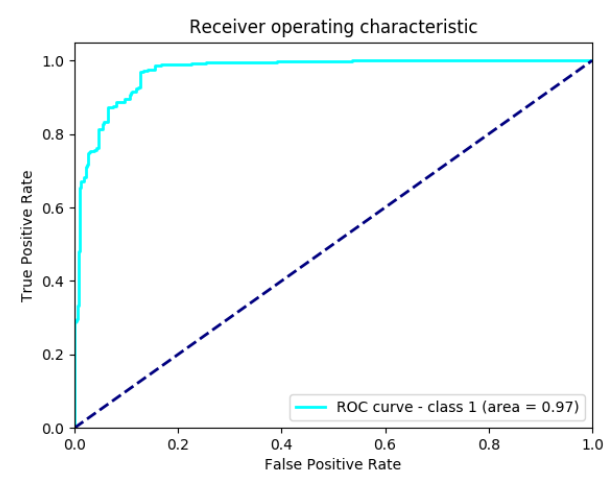

(a)

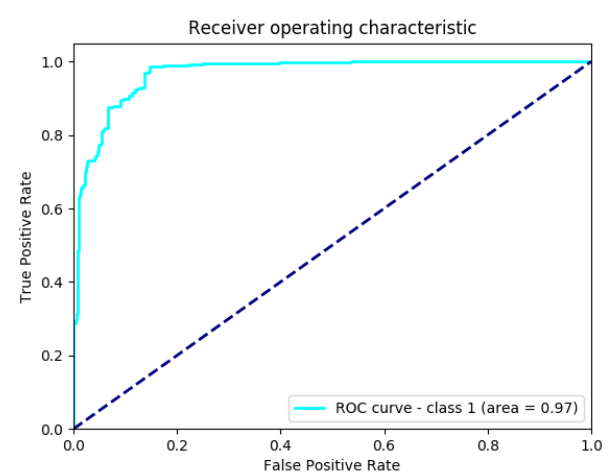

(b)

Figure A.26: N-grams with Random Forest as classifier (a) Random Forest with Trees $=200$; (b) Random Forest with Trees $=300$; 